\title{
COMPACT MANIFOLDS WITH COMPUTABLE BOUNDARIES
}

\author{
ZVONKO ILJAZOVIĆ \\ Department of Mathematics, Faculty of Science, University of Zagreb, Croatia \\ e-mail address: zilj@math.hr
}

\begin{abstract}
We investigate conditions under which a co-computably enumerable closed set in a computable metric space is computable and prove that in each locally computable computable metric space each co-computably enumerable compact manifold with computable boundary is computable. In fact, we examine the notion of a semi-computable compact set and we prove a more general result: in any computable metric space each semicomputable compact manifold with computable boundary is computable. In particular, each semi-computable compact (boundaryless) manifold is computable.
\end{abstract}

\section{INTRODUCTION}

If $f: \mathbb{R} \rightarrow \mathbb{R}$ is a computable function and if for some $a<b$ we have $f(a)<0$ and $f(b)>0$, then there exists a computable number $c$ between $a$ and $b$ such that $f(c)=0$ [13. In general, however, we cannot conclude that a computable function which has a zero-point has a computable zero-point: there exists a computable function $f: \mathbb{R} \rightarrow \mathbb{R}$ which has zero-points, but none of them is computable [12]. However, it is known that if a computable function $f: \mathbb{R}^{m} \rightarrow \mathbb{R}, m \geq 1$, has an isolated zero-point, then that point must be computable. This raises the following question: under what conditions a computable function $f: \mathbb{R}^{m} \rightarrow \mathbb{R}$ has a computable zero-point, or, even better, under what conditions the set $f^{-1}(\{0\})$ of all zero points of $f$ is computable?

A closed subset of $\mathbb{R}^{m}$ is computable if it can be effectively approximated by a finite set of points with rational coordinates with arbitrary precision on an arbitrary bounded region of $\mathbb{R}^{m}$. Each nonempty computable set contains computable points, in fact they are dense in it. On the other hand, if $S \subseteq \mathbb{R}^{m}$, then $S$ is equal to $f^{-1}(\{0\})$ for some computable function $f: \mathbb{R}^{m} \rightarrow \mathbb{R}$ if and only if the complement of $S$ can be effectively covered by open balls. A closed subset of $\mathbb{R}^{m}$ is called co-computably enumerable (co-c.e.) if its complement can be effectively covered by open balls. So the question under what conditions a set of the form $f^{-1}(\{0\})$ is computable can be restated in the following way: under what conditions a co-c.e. set is computable, i.e. under what conditions the implication

$S$ co-computably enumerable closed set $\Rightarrow S$ computable closed set

2012 ACM CCS: [Theory of computation]: Models of computation-Computability-Recursive functions.

Key words and phrases: computable metric space, computable set, co-c.e. set, semi-computable compact set, manifold with boundary.

닌 
holds?

The implication (1.1) does not hold in general, moreover, as mentioned, there exists a nonempty co-c.e. subset of $\mathbb{R}$ which contains no computable points. Such a set cannot be connected (otherwise it would consist of a single point or it would contain an open interval). It is easy to construct a connected co-c.e. subset of $\mathbb{R}^{2}$ which contains no computable points, but it is an interesting question is it possible to achieve that such a set is even simply connected [11]. Kihara [8] recently showed that such a set does exist.

Regarding the implication (1.1), it fails to be true even for sets which are very simple from the topological viewpoint: in each $\mathbb{R}^{m}$ there exists a line segment which is co-c.e., but not computable [9]. However, there are certain conditions under which this implication holds. In [6] it was proved that (1.1) holds when $S$ is a circularly chainable continuum or a continuum chainable from $a$ to $b$, where $a$ and $b$ are computable points. Brattka [1] gave some results concerning (1.1) in the case when $S$ is the graph of a continuous function. Miller [9] proved that (1.1) holds whenever $S \subseteq \mathbb{R}^{m}$ is a topological sphere (i.e. homeomorphic to the unit sphere $S^{n} \subseteq \mathbb{R}^{n+1}$ for some $n$ ) or $S$ is homeomorphic to the closed unit ball $B^{n} \subseteq \mathbb{R}^{n}$ for some $n$ (i.e. $S$ is an $n$-cell) by a homeomorphism $f: B^{n} \rightarrow S$ such that $f\left(S^{n-1}\right)$ is co-c.e. set in $\mathbb{R}^{m}$. Furthermore, by [7], these results hold not just in $\mathbb{R}^{m}$, but also in any computable metric space which is locally computable.

This, for example, means that each co-c.e. arc in $\mathbb{R}^{m}$ is computable if its endpoints are computable. Hence the computability of endpoints implies the computability of the whole arc. And if we have a co-c.e. cell, then the computability of its boundary sphere implies the computability of the whole cell. Since arcs and cells are examples of spaces known as manifolds with boundary, the question which arises here is what can be said about computability of such spaces, i.e. if we have a co-c.e. manifold with boundary, does the computability of its boundary imply the computability of the whole manifold?

In this paper we answer this question. We prove that if $(X, d, \alpha)$ is a locally computable computable metric space and if $S$ is a co-c.e. closed set in $(X, d, \alpha)$ which is, as a subspace of $(X, d)$, a compact manifold with boundary, then the computability of $\partial S$ implies the computability of $S$. This in particular means that a co-c.e. set $S$ is computable if it is a (boundaryless) manifold. A manifold with boundary is a topological space in which each point is contained in some open set which is homeomorphic to Euclidean space $\mathbb{R}^{n}$ or Euclidean half-space $\left\{\left(x_{1}, \ldots, x_{n}\right) \mid x_{n} \geq 0\right\}$. If $X$ is a manifold with boundary, then the boundary $\partial X$ of $X$ consists of all points $x \in X$ such that none of the open sets containing $x$ is homeomorphic to Euclidean space. If $X$ is a manifold with boundary and $\partial X=\emptyset$, than we simply say that $X$ is a manifold.

For example, the unit sphere $S^{n}$ is a manifold and the unit ball $B^{n}$ is a manifold with boundary, its boundary is $S^{n-1}$. Moreover, each topological sphere is a manifold and if $f: B^{n} \rightarrow X$ is a homeomorphism, then $X$ is a manifold with boundary and $\partial X=f\left(S^{n-1}\right)$.

In fact, we will prove a result which turns out to be more general: in any computable metric space $(X, d, \alpha)$ the implication

$$
S \text { semi-computable compact set } \Rightarrow S \text { computable compact set }
$$

holds if $S$ is a compact manifold with computable boundary. That $S$ is a semi-computable compact set means that $S$ is compact and we can effectively enumerate all rational open sets which cover $S$. In other words, we will prove that if $S$ is semi-computable compact manifold with boundary in $(X, d, \alpha)$ then the following implication holds:

$$
\partial S \text { computable } \Rightarrow S \text { computable. }
$$


In order to prove this, the central notion will be the notion of computability up to some set. Using techniques from [6] and [7] (in particular using $n$-dimensional chains) we will see that in each semi-computable compact manifold with computable boundary each point has a neighborhood which is computable up to the manifold. This fact will then imply that a semi-computable compact manifold with computable boundary is computable.

In Section 2 we give some basic notions and in Section 3 we examine semi-computable compact sets. In Section 4 we introduce the notion of computability up to a set. In Section 5 we examine $n$-chains and prove that in each semi-computable compact manifold with computable boundary each point has a neighborhood which is computable up to the manifold (Theorem 5.6. In Section 6 we prove that semi-computable compact manifolds with computable boundaries are computable.

\section{BASIC NOTIONS AND TECHNiques}

If $X$ is a set, let $\mathcal{P}(X)$ denote the set of all subsets of $X$.

For $m \in \mathbb{N}$ let $\mathbb{N}_{m}=\{0, \ldots, m\}$. For $n \geq 1$ let

$$
\mathbb{N}_{m}^{n}=\left\{\left(x_{1}, \ldots, x_{n}\right) \mid x_{1}, \ldots, x_{n} \in \mathbb{N}_{m}\right\} .
$$

We say that a function $\Phi: \mathbb{N}^{k} \rightarrow \mathcal{P}\left(\mathbb{N}^{n}\right)$ is computable if the function $\bar{\Phi}: \mathbb{N}^{k+n} \rightarrow \mathbb{N}$ defined by

$$
\bar{\Phi}(x, y)=\chi_{\Phi(x)}(y)
$$

$x \in \mathbb{N}^{k}, y \in \mathbb{N}^{n}$, is computable (i.e. recursive). Here $\chi_{S}: \mathbb{N}^{n} \rightarrow\{0,1\}$ denotes the characteristic function of $S \subseteq \mathbb{N}^{n}$. A function $\Phi: \mathbb{N}^{k} \rightarrow \mathcal{P}\left(\mathbb{N}^{n}\right)$ is said to be computably bounded if there exists a computable function $\varphi: \mathbb{N}^{k} \rightarrow \mathbb{N}$ such that $\Phi(x) \subseteq \mathbb{N}_{\varphi(x)}^{n}$ for all $x \in \mathbb{N}^{k}$.

We say that a function $\Phi: \mathbb{N}^{k} \rightarrow \mathcal{P}\left(\mathbb{N}^{n}\right)$ is c.c.b. if $\Phi$ is computable and computably bounded.

\section{Proposition 2.1.}

(1) If $\Phi, \Psi: \mathbb{N}^{k} \rightarrow \mathcal{P}\left(\mathbb{N}^{n}\right)$ are c.c.b. functions, then the function $\mathbb{N}^{k} \rightarrow \mathcal{P}\left(\mathbb{N}^{n}\right), x \mapsto$ $\Phi(x) \cup \Psi(x)$ is c.c.b.

(2) If $\Phi, \Psi: \mathbb{N}^{k} \rightarrow \mathcal{P}\left(\mathbb{N}^{n}\right)$ are c.c.b. functions, then the sets $\left\{x \in \mathbb{N}^{k} \mid \Phi(x)=\Psi(x)\right\}$ and $\left\{x \in \mathbb{N}^{k} \mid \Phi(x) \subseteq \Psi(x)\right\}$ are decidable.

(3) Let $\Phi: \mathbb{N}^{k} \rightarrow \mathcal{P}\left(\mathbb{N}^{n}\right)$ and $\Psi: \mathbb{N}^{n} \rightarrow \mathcal{P}\left(\mathbb{N}^{m}\right)$ be c.c.b. functions. Let $\Lambda: \mathbb{N}^{k} \rightarrow \mathcal{P}\left(\mathbb{N}^{m}\right)$ be defined by

$$
\Lambda(x)=\bigcup_{z \in \Phi(x)} \Psi(z)
$$

$x \in \mathbb{N}^{k}$. Then $\Lambda$ is a c.c.b. function.

(4) Let $\Phi: \mathbb{N}^{k} \rightarrow \mathcal{P}\left(\mathbb{N}^{n}\right)$ be c.c.b. and let $T \subseteq \mathbb{N}^{n}$ be c.e. Then the set $S=\left\{x \in \mathbb{N}^{k} \mid\right.$ $\Phi(x) \subseteq T\}$ is c.e. 
A function $F: \mathbb{N}^{k} \rightarrow \mathbb{Q}$ is called computable if there exist computable functions $a, b, c:$ $\mathbb{N}^{k} \rightarrow \mathbb{N}$ such that

$$
F(x)=(-1)^{c(x)} \frac{a(x)}{b(x)+1}
$$

for each $x \in \mathbb{N}^{k}$. A number $x \in \mathbb{R}$ is said to be computable if there exists a computable function $g: \mathbb{N} \rightarrow \mathbb{Q}$ such that $|x-g(i)|<2^{-i}$ for each $i \in \mathbb{N}[14$.

By a computable function $\mathbb{N}^{k} \rightarrow \mathbb{R}$ we mean a function $f: \mathbb{N}^{k} \rightarrow \mathbb{R}$ for which there exists a computable function $F: \mathbb{N}^{k+1} \rightarrow \mathbb{Q}$ such that

$$
|f(x)-F(x, i)|<2^{-i}
$$

for all $x \in \mathbb{N}^{k}$ and $i \in \mathbb{N}$.

In the following proposition we state some facts about computable functions $\mathbb{N}^{k} \rightarrow \mathbb{R}$.

\section{Proposition 2.2.}

(1) If $f, g: \mathbb{N}^{k} \rightarrow \mathbb{R}$ are computable, then $f+g, f-g, \sqrt{|f|}: \mathbb{N}^{k} \rightarrow \mathbb{R}$ are computable.

(2) If $f: \mathbb{N}^{k} \rightarrow \mathbb{R}$ and $F: \mathbb{N}^{k+1} \rightarrow \mathbb{R}$ are functions such that $F$ is computable and $|f(x)-F(x, i)|<2^{-i}$ for each $x \in \mathbb{N}^{k}$ and $i \in \mathbb{N}$, then $f$ is computable.

(3) If $f, g: \mathbb{N}^{k} \rightarrow \mathbb{R}$ are computable functions, then the set $\left\{x \in \mathbb{N}^{k} \mid f(x)>g(x)\right\}$ is c.e.

(4) If $f: \mathbb{N}^{k} \rightarrow \mathbb{R}$ is a computable function and $\Phi: \mathbb{N}^{n} \rightarrow \mathcal{P}\left(\mathbb{N}^{k}\right)$ is a c.c.b. function such that $\Phi(x) \neq \emptyset$ for each $x \in \mathbb{N}^{n}$, then the functions $g, h: \mathbb{N}^{n} \rightarrow \mathbb{R}$ defined by

$$
g(x)=\max _{y \in \Phi(x)} f(y), h(x)=\min _{y \in \Phi(x)} f(y)
$$

are computable.

2.1. Computable metric spaces. A tuple $(X, d, \alpha)$ is said to be a computable metric space if $(X, d)$ is a metric space and $\alpha: \mathbb{N} \rightarrow X$ is a sequence dense in $(X, d)$ (i.e. a sequence whose range is dense in $(X, d))$ such that the function $\mathbb{N}^{2} \rightarrow \mathbb{R}$,

$$
(i, j) \mapsto d\left(\alpha_{i}, \alpha_{j}\right)
$$

is computable (we use notation $\alpha=\left(\alpha_{i}\right)$ ).

If $(X, d, \alpha)$ is a computable metric space, then a sequence $\left(x_{i}\right)$ in $X$ is said to be computable in $(X, d, \alpha)$ if there exists a computable function $F: \mathbb{N}^{2} \rightarrow \mathbb{N}$ such that

$$
d\left(x_{i}, \alpha_{F(i, k)}\right)<2^{-k}
$$

for all $i, k \in \mathbb{N}$. A point $a \in X$ is said to be computable in $(X, d, \alpha)$ if there exists a computable function $f: \mathbb{N} \rightarrow \mathbb{N}$ such that $d\left(a, \alpha_{f(k)}\right)<2^{-k}$ for each $k \in \mathbb{N}$.

The points $\alpha_{0}, \alpha_{1}, \ldots$ are called rational points. If $i \in \mathbb{N}$ and $q \in \mathbb{Q}, q>0$, then we say that $B\left(\alpha_{i}, q\right)$ is an (open) rational ball and $\widehat{B}\left(\alpha_{i}, q\right)$ is a closed rational ball. Here, for $x \in X$ and $r>0$, we denote by $B(x, r)$ the open ball of radius $r$ centered at $x$ and by $\widehat{B}(x, r)$ the corresponding closed ball, i.e. $B(x, r)=\{y \in X \mid d(x, y)<r\}$, $\widehat{B}(x, r)=\{y \in X \mid d(x, y) \leq r\}$.

If $B_{1}, \ldots, B_{n}, n \geq 1$, are open rational balls, then the union $B_{1} \cup \cdots \cup B_{n}$ will be called a rational open set. 
Example 2.3. If $\alpha: \mathbb{N} \rightarrow \mathbb{R}^{n}$ is a computable function (in the sense that the component functions of $\alpha$ are computable) whose image is dense in $\mathbb{R}^{n}$ and $d$ is the Euclidean metric on $\mathbb{R}^{n}$, then $\left(\mathbb{R}^{n}, d, \alpha\right)$ is a computable metric space (Proposition 2.2). A sequence $\left(x_{i}\right)$ is computable in this computable metric space if and only if $\left(x_{i}\right)$ is a computable sequence in $\mathbb{R}^{n}$ and $\left(x_{1}, \ldots, x_{n}\right) \in \mathbb{R}^{n}$ is a computable point in this space if and only if $x_{1}, \ldots, x_{n}$ are computable numbers.

2.2. Effective enumerations. Let $(X, d, \alpha)$ be a computable metric space. Let $q: \mathbb{N} \rightarrow \mathbb{Q}$ be some fixed computable function whose image is $\mathbb{Q} \cap\langle 0, \infty\rangle$ and let $\tau_{1}, \tau_{2}: \mathbb{N} \rightarrow \mathbb{N}$ be some fixed computable functions such that $\left\{\left(\tau_{1}(i), \tau_{2}(i)\right) \mid i \in \mathbb{N}\right\}=\mathbb{N}^{2}$. Let $\left(\lambda_{i}\right)_{i \in \mathbb{N}}$ be the sequence of points in $X$ defined by $\lambda_{i}=\alpha_{\tau_{1}(i)}$ and let $\left(\rho_{i}\right)_{i \in \mathbb{N}}$ be the sequence of rational numbers defined by $\rho_{i}=q_{\tau_{2}(i)}$. For $i \in \mathbb{N}$ we define

$$
I_{i}=B\left(\lambda_{i}, \rho_{i}\right), \widehat{I}_{i}=\widehat{B}\left(\lambda_{i}, \rho_{i}\right) .
$$

The sequences $\left(I_{i}\right)$ and $\left(\widehat{I}_{i}\right)$ represent effective enumerations of all open rational balls and all closed rational balls.

Now we want to define some effective enumeration of all rational open sets.

Let $\sigma: \mathbb{N}^{2} \rightarrow \mathbb{N}$ and $\eta: \mathbb{N} \rightarrow \mathbb{N}$ be some fixed computable functions with the following property: $\{(\sigma(j, 0), \ldots, \sigma(j, \eta(j))) \mid j \in \mathbb{N}\}$ is the set of all finite sequences in $\mathbb{N}$ (excluding the empty sequence), i.e. the set $\left\{\left(a_{0}, \ldots, a_{n}\right) \mid n \in \mathbb{N}, a_{0}, \ldots, a_{n} \in \mathbb{N}\right\}$. Such functions, for instance, can be defined using the Cantor pairing function. We use the following notation: $(j)_{i}$ instead of $\sigma(j, i)$ and $\bar{j}$ instead of $\eta(j)$. Hence

$$
\left\{\left((j)_{0}, \ldots,(j)_{\bar{j}}\right) \mid j \in \mathbb{N}\right\}
$$

is the set of all finite sequences in $\mathbb{N}$. For $j \in \mathbb{N}$ let $[j]$ be defined by

$$
[j]=\left\{(j)_{i} \mid 0 \leq i \leq \bar{j}\right\} .
$$

Note that the function $\mathbb{N} \rightarrow \mathcal{P}(\mathbb{N}), j \mapsto[j]$, is c.c.b.

For $j \in \mathbb{N}$ we define

$$
J_{j}=\bigcup_{i \in[j]} I_{i}
$$

Then $\left(J_{j}\right)$ is an effective enumeration of all rational open sets.

For $i \in \mathbb{N}$ we define $\Lambda_{i}=\alpha([i])$, hence

$$
\Lambda_{i}=\left\{\alpha_{(i)_{0}}, \ldots, \alpha_{(i)_{\bar{i}}}\right\} .
$$

The sequence $\left(\Lambda_{i}\right)$ is an effective enumeration of all finite nonempty sets of rational points.

2.3. Formal diameter and formal disjointness. In Euclidean space $\mathbb{R}^{n}$ we can effectively calculate the diameter of the finite union of rational balls. However, in a general computable metric space the function $\mathbb{N} \rightarrow \mathbb{R}, j \mapsto \operatorname{diam}\left(J_{j}\right)$, need not be computable. For that reason we are going to use the notion of the formal diameter. Let $(X, d)$ be a metric space and $x_{0}, \ldots, x_{k} \in X, r_{0}, \ldots, r_{k} \in \mathbb{R}_{+}$. The formal diameter associated to the finite sequence $\left(x_{0}, r_{0}\right), \ldots,\left(x_{k}, r_{k}\right)$ is the number $D \in \mathbb{R}$ defined by

$$
D=\max _{0 \leq v, w \leq k} d\left(x_{v}, x_{w}\right)+2 \max _{0 \leq v \leq k} r_{v} .
$$

It follows from this definition that $\operatorname{diam}\left(B\left(x_{0}, r_{0}\right) \cup \cdots \cup B\left(x_{k}, r_{k}\right)\right) \leq D$. 
Let $(X, d, \alpha)$ be a computable metric space. We define the function fdiam $: \mathbb{N} \rightarrow \mathbb{R}$ in the following way. For $j \in \mathbb{N}$ the number $\operatorname{fdiam}(j)$ is the formal diameter associated to the finite sequence

$$
\left(\lambda_{(j)_{0}}, \rho_{(j)_{0}}\right), \ldots,\left(\lambda_{(j)_{\bar{j}}}, \rho_{(j)_{\bar{j}}}\right) .
$$

Clearly $\operatorname{diam}\left(J_{j}\right) \leq \operatorname{fdiam}(j)$ for each $j \in \mathbb{N}$.

Let $i, j \in \mathbb{N}$. We say that $I_{i}$ and $I_{j}$ are formally disjoint if

$$
d\left(\lambda_{i}, \lambda_{j}\right)>\rho_{i}+\rho_{j} .
$$

Note that we define this as a relation between the numbers $i$ and $j$, not the sets $I_{i}$ and $I_{j}$ (it is possible that for some $i, i^{\prime}, j, j^{\prime}$ we have that $I_{i}$ and $I_{j}$ are formally disjoint and $I_{i}=I_{i^{\prime}}$, $I_{j}=I_{j^{\prime}}$, but $I_{i^{\prime}}$ and $I_{j^{\prime}}$ are not formally disjoint, for example such a situation can occur if $d$ is the discrete metric on $X$ ). If $I_{i}$ and $I_{j}$ are formally disjoint, then $I_{i} \cap I_{j}=\emptyset$.

Let $i, j \in \mathbb{N}$. We say that $J_{i}$ and $J_{j}$ are formally disjoint if $I_{k}$ and $I_{l}$ are formally disjoint for all $k \in[i]$ and $l \in[j]$. Clearly, if $J_{i}$ and $J_{j}$ are formally disjoint, then $J_{i} \cap J_{j}=\emptyset$.

\section{Proposition 2.4.}

(1) The function fdiam $: \mathbb{N} \rightarrow \mathbb{R}$ is computable.

(2) The set $\left\{(i, j) \in \mathbb{N}^{2} \mid J_{i}\right.$ and $J_{j}$ are formally disjoint $\}$ is c.e.

Proof. This follows from Proposition 2.1 and Proposition 2.2 (for details see [6], Proposition 13 and Proposition 8).

2.4. Computable sets. Let $(X, d, \alpha)$ be a computable metric space. We say that $S$ is a computably enumerable closed set in $(X, d, \alpha)$ if $S$ is a closed subset of $(X, d)$ and if

$$
\left\{i \in \mathbb{N} \mid S \cap I_{i} \neq \emptyset\right\}
$$

is a c.e. subset of $\mathbb{N}$. A closed subset of $(X, d)$ is said to be co-computably enumerable closed set in $(X, d, \alpha)$ if there exists a computable function $f: \mathbb{N} \rightarrow \mathbb{N}$ such that

$$
X \backslash S=\bigcup_{i \in \mathbb{N}} I_{f(i)}
$$

It is easy to see that these definitions do not depend on functions $\tau_{1}, \tau_{2}$ and $q$. We say that $S$ is a computable closed set in $(X, d, \alpha)$ if $S$ is a computably enumerable closed set and a co-computably enumerable closed set [2, 15].

We say that $K$ is a computable compact set in $(X, d, \alpha)$ if $K$ is a compact set in $(X, d)$, the set $\left\{j \in \mathbb{N} \mid K \subseteq J_{j}\right\}$ is c.e. and $K$ is a c.e. closed set in $(X, d, \alpha)$.

2.5. Hausdorff metric. Let $(X, d, \alpha)$ be a computable metric space. Let $A, B \subseteq X$ and $\varepsilon>0$. We write $A \prec_{\varepsilon} B$ if for each $a \in A$ there exists $b \in B$ such that $d(a, b)<\varepsilon$. We write

$$
A \approx_{\varepsilon} B
$$

if $A \prec_{\varepsilon} B$ and $B \prec_{\varepsilon} A$. Note that $A \prec_{\varepsilon} B$ and $B \prec_{\varepsilon^{\prime}} C$ imply $A \prec_{\varepsilon+\varepsilon^{\prime}} C$. Also note that $A \prec_{\varepsilon} B$ and $A^{\prime} \prec_{\varepsilon} B^{\prime}$ imply $A \cup A^{\prime} \prec_{\varepsilon} B \cup B^{\prime}$.

Let $\mathcal{H}$ be the set of all nonempty compact subsets of $(X, d)$. The Hausdorff metric on $\mathcal{H}$ is the metric $\varrho$ on $\mathcal{H}$ defined by

$$
\varrho(A, B)=\inf \left\{\varepsilon>0 \mid A \approx_{\varepsilon} B\right\} .
$$


If $A, B \in \mathcal{H}$ and $\varepsilon>0$, then

$$
A \prec_{\varepsilon} B \Leftrightarrow d(a, B)<\varepsilon \text { for each } a \in A \Leftrightarrow \max _{a \in A} d(a, B)<\varepsilon
$$

(the last equivalence follows from the fact that the continuous function $X \rightarrow \mathbb{R}, x \mapsto d(x, B)$ attains its maximum on $A$ ). Therefore, for all $A, B \in \mathcal{H}$ we have

$$
\varrho(A, B)=\max \left\{\max _{a \in A} d(a, B), \max _{b \in B} d(b, A)\right\} .
$$

Also note that for $A, B \in \mathcal{H}$ and $\varepsilon>0$ we have $\varrho(A, B)<\varepsilon$ if and only if $A \approx_{\varepsilon} B$.

Proposition 2.5. Let $(X, d, \alpha)$ be a computable metric space. The function $\mathbb{N}^{2} \rightarrow \mathbb{R}$, $(i, j) \mapsto \varrho\left(\Lambda_{i}, \Lambda_{j}\right)$ is computable.

Proof. This follows from 2.2 and Proposition 2.2(4).

It is easy to conclude that the sequence $\left(\Lambda_{i}\right)$ is dense in the metric space $(\mathcal{H}, \varrho)$. Therefore, the triple $(\mathcal{H}, \varrho, \Lambda)$ is a computable metric space. The following proposition says that computable points in this space are exactly nonempty computable compact sets in $(X, d, \alpha)$ (see also Theorem 4.12. in [2]).

Proposition 2.6. Let $(X, d, \alpha)$ be a computable metric space and let $K$ be a nonempty compact set in $(X, d)$. Then $K$ is a computable compact set in $(X, d, \alpha)$ if and only if there exists a computable function $f: \mathbb{N} \rightarrow \mathbb{N}$ such that

$$
\varrho\left(K, \Lambda_{f(k)}\right)<2^{-k}
$$

for each $k \in \mathbb{N}$.

Proof. Suppose $K$ is a computable compact set. Then the set $\left\{i \in \mathbb{N} \mid I_{i} \cap K \neq \emptyset\right\}$ is c.e. and we conclude from Proposition 2.1 (4) that the set $\left\{j \in \mathbb{N} \mid I_{i} \cap K \neq \emptyset\right.$ for each $\left.i \in[j]\right\}$ is c.e. Similarly, using also Proposition 2.2(3), we get that the set

$$
\left\{(j, k) \in \mathbb{N}^{2} \mid \rho_{i}<2^{-k} \text { for each } i \in[j]\right\}
$$

is c.e. Therefore the set

$$
\Omega=\left\{(j, k) \in \mathbb{N}^{2} \mid K \subseteq J_{j}, I_{i} \cap K \neq \emptyset \text { and } \rho_{i}<2^{-k} \text { for each } i \in[j]\right\}
$$

is c.e. Since for each $k \in \mathbb{N}$ there exist $j \in \mathbb{N}$ such that $(j, k) \in \Omega$, there exists a computable function $\varphi: \mathbb{N} \rightarrow \mathbb{N}$ such that $(\varphi(k), k) \in \Omega$ for each $k \in \mathbb{N}$. Since the function $k \mapsto[k]$ is c.c.b. and each nonempty subset of $\mathbb{N}$ is equal to $[k]$ for some $k \in \mathbb{N}$, Proposition 2.1 implies that there exists a computable function $f: \mathbb{N} \rightarrow \mathbb{N}$ such that $[f(k)]=\left\{\tau_{1}(i) \mid i \in[\varphi(k)]\right\}$ for each $k \in \mathbb{N}$. Then

and it follows that $\varrho\left(K, \Lambda_{f(k)}\right)<2^{-k}$.

$$
\Lambda_{f(k)}=\left\{\lambda_{i} \mid i \in[\varphi(k)]\right\}
$$

Conversely, suppose that (2.3) holds for some computable function $f: \mathbb{N} \rightarrow \mathbb{N}$. We want to prove that $K$ is a computable compact set.

If $x, y \in X$ and $r, s>0$, we will say that $(y, s)$ is formally contained in $(x, r)$ and write $(y, s) \subseteq_{F}(x, r)$ if $d(x, y)+s<r$. Note that $(y, s) \subseteq_{F}(x, r)$ implies $B(y, s) \subseteq B(x, r)$.

Suppose that $K \subseteq J_{j}$ for some $j \in \mathbb{N}$. Using compactness of $K$ it is not hard to conclude (see Lemma 19 in [6]) that there exists $\mu>0$ with property that for each $x \in K$ there exists $i \in[j]$ such that $(x, 2 \mu) \subseteq_{F}\left(\lambda_{i}, \rho_{i}\right)$. Choose $k \in \mathbb{N}$ so that $2^{-k}<\mu$. Let $l \in[f(k)]$. Since 
(2.3) holds, there exists $x \in K$ such that $d\left(\alpha_{l}, x\right)<\mu$. On the other hand, there exists $i \in[j]$ such that $(x, 2 \mu) \subseteq_{F}\left(\lambda_{i}, \rho_{i}\right)$. It follows $\left(\alpha_{l}, \mu\right) \subseteq_{F}\left(\lambda_{i}, \rho_{i}\right)$. Hence

$$
(\forall l \in[f(k)]) \quad(\exists i \in[j])\left(\alpha_{l}, 2^{-k}\right) \subseteq_{F}\left(\lambda_{i}, \rho_{i}\right) .
$$

However, if $j, k \in \mathbb{N}$ are such that (2.4) holds, then we have $K \subseteq J_{j}$. Namely, for each $x \in K$ by 2.3 there exists $l \in[f(k)]$ such that $d\left(x, \alpha_{l}\right)<2^{-k}$ and, by $(2.4)$, there exists $i \in[j]$ such that $d\left(\alpha_{l}, \lambda_{i}\right)+2^{-k}<\rho_{i}$ from which we get $d\left(x, \lambda_{i}\right)<\rho_{i}$. Hence $x \in J_{j}$.

Therefore $K \subseteq J_{j}$ if and only if there exists $k \in \mathbb{N}$ such that (2.4) holds. Since the set of all $(j, k) \in \mathbb{N}^{2}$ such that (2.4) holds is c.e. (which follows from Proposition 2.2 and Proposition 2.1), the set $\left\{j \in \mathbb{N} \mid K \subseteq J_{j}\right\}$ is c.e.

Let $i \in \mathbb{N}$. Then using (2.3) we conclude that

$$
K \cap I_{i} \neq \emptyset \Leftrightarrow(\exists x \in K) d\left(x, \lambda_{i}\right)<\rho_{i} \Leftrightarrow(\exists k \in \mathbb{N})(\exists l \in[f(k)]) d\left(\alpha_{l}, \lambda_{i}\right)+2^{-k}<\rho_{i}
$$

and it follows from Proposition 2.2 that $K$ is a c.e. closed set.

\section{Semi-Computable compact Sets}

Let $(X, d, \alpha)$ be a computable metric space. We say that $K$ is a semi-computable compact set in $(X, d, \alpha)$ if $K$ is a compact set in $(X, d)$ and if the set $\left\{j \in \mathbb{N} \mid K \subseteq J_{j}\right\}$ is c.e. Hence $K$ is a computable compact set if and only if $K$ is a semi-computable compact set and $K$ is a c.e. closed set.

Proposition 3.1. Let $(X, d, \alpha)$ be a computable metric space. If $S$ is a semi-computable compact set in $(X, d, \alpha)$, then $S$ is a co-c.e. closed set in $(X, d, \alpha)$.

Proof. Let $L$ be the set of all $l \in \mathbb{N}$ for which there exists $j \in \mathbb{N}$ such that $S \subseteq J_{j}$ and such that $I_{l}$ is formally disjoint with $I_{i}$ for each $i \in[j]$. Since the set $\left\{(l, i) \in \mathbb{N}^{2} \mid I_{l}\right.$ and $I_{i}$ are formally disjoint $\}$ is c.e. by Proposition $2.2, L$ is c.e. Clearly $\bigcup_{l \in L} I_{l} \subseteq X \backslash S$.

On the other hand, let $x \in X \backslash S$. Then, since $S$ is compact, there exists $\mu>0$ such that $d(x, s)>4 \mu$ for each $s \in S$. Compactness of $S$ furthermore implies that there exists $j \in \mathbb{N}$ such that $S \subseteq J_{j}$ and such that $\rho_{i}<\mu$ and $I_{i} \cap S \neq \emptyset$ for each $i \in[j]$. Choose $l \in \mathbb{N}$ so that $x \in I_{l}$ and $\rho_{l}<\mu$. It is straightforward to check that $I_{l}$ and $I_{i}$ are formally disjoint for each $i \in[j]$. Hence $l \in L$ and this proves that $X \backslash S \subseteq \bigcup_{l \in L} I_{l}$, hence $X \backslash S=\bigcup_{l \in L} I_{l}$. $\square$

The preceding proposition implies that each computable compact set is computable closed set.

A computable metric space $(X, d, \alpha)$ is locally computable [1] if for each compact set $A$ in $(X, d)$ there exists a computable compact set $K$ in $(X, d, \alpha)$ such that $A \subseteq K$. For example, the computable metric space $\left(\mathbb{R}^{n}, d, \alpha\right)$ from Example 2.3 is locally computable (this can be deduced from Proposition 2.6).

Proposition 3.2. Let $(X, d, \alpha)$ be a computable metric space which is locally computable. Let $S$ be a co-c.e. closed set in $(X, d, \alpha)$ which is compact. Then $S$ is a semi-computable compact set in $(X, d, \alpha)$.

Proof. Since $S$ is co-c.e., there exists a computable function $f: \mathbb{N} \rightarrow \mathbb{N}$ such that

$$
X \backslash S=\bigcup_{n \in \mathbb{N}} J_{f(n)}
$$


and $J_{f(n)} \subseteq J_{f(n+1)}$ for each $n \in \mathbb{N}$. Furthermore, there exists a computable compact set $K$ such that $S \subseteq K$.

Let $j \in \mathbb{N}$. If $S \subseteq J_{j}$, then $K \backslash J_{j}$ is a compact subset of $X \backslash S$ and therefore there exists $n \in \mathbb{N}$ such that $K \backslash J_{j} \subseteq J_{f(n)}$. We have the following conclusion:

$$
S \subseteq J_{j} \Leftrightarrow(\exists n \in \mathbb{N}) K \subseteq J_{j} \cup J_{f(n)} .
$$

By Proposition 2.1 $(1)$ the function $\mathbb{N}^{2} \rightarrow \mathcal{P}(\mathbb{N}),(j, n) \mapsto[j] \cup[f(n)]$ is c.c.b. and therefore by claim (2) of the same proposition there exists a computable function $g: \mathbb{N}^{2} \rightarrow \mathbb{N}$ such that $[j] \cup[f(n)]=[g(j, n)]$ for all $j, n \in \mathbb{N}$. Hence $J_{j} \cup J_{f(n)}=J_{g(j, n)}$ and the set of all $(j, n) \in \mathbb{N}^{2}$ such that $K \subseteq J_{g(j, n)}$ is c.e. since $K$ is semi-computable. Now (3.1) implies that $S$ is semi-computable. cation

In this paper we will prove that in every computable metric space $(X, d, \alpha)$ the impli-

$$
S \text { semi-computable compact set } \Rightarrow S \text { computable compact set }
$$

holds for each compact manifold $S$ with computable boundary. Using this and Proposition 3.2 we conclude that if $(X, d, \alpha)$ is locally computable, then for every compact manifold $S$ with computable boundary the following implication holds:

$$
S \text { co-c.e. closed } \Rightarrow S \text { computable closed. }
$$

Lemma 3.3. Let $(X, d, \alpha)$ be a computable metric space and let $S$ be a semi-computable compact set in this space. Let $m \in \mathbb{N}$. Then $S \backslash J_{m}$ is a semi-computable compact set.

Proof. Let $j \in \mathbb{N}$. Then $S \backslash J_{m} \subseteq J_{j}$ if and only if $S \subseteq J_{j} \cup J_{m}$ and it follows that $S \backslash J_{m}$ is semi-computable (similarly as in the proof of the previous proposition).

\section{Computability up to a Set}

Let $(X, d, \alpha)$ be a computable metric space. Let $A, B \subseteq X, A \subseteq B$. We say that $A$ is computable up to $B$ if there exists a computable function $f: \mathbb{N} \rightarrow \mathbb{N}$ such that

$$
A \prec_{2^{-k}} \Lambda_{f(k)} \text { and } \Lambda_{f(k)} \prec_{2^{-k}} B
$$

for each $k \in \mathbb{N}$. Using Proposition 2.6 we conclude that for each nonempty compact subset $A$ of $(X, d)$ the following equivalence holds:

$A$ is a computable compact set $\Leftrightarrow A$ is computable up to $A$.

Proposition 4.1. Let $(X, d, \alpha)$ be a computable metric space. Suppose $A, B$ and $S$ are subsets of $X$ such that $A \subseteq S$ and $B \subseteq S$ and such that $A$ and $B$ are computable up to $S$. Then $A \cup B$ is computable up to $S$.

Proof. Let $f, g: \mathbb{N} \rightarrow \mathbb{N}$ be computable functions such that $A \prec_{2^{-k}} \Lambda_{f(k)} \prec_{2^{-k}} S$ and $B \prec_{2^{-k}} \Lambda_{g(k)} \prec_{2^{-k}} S$ for each $k \in \mathbb{N}$. Then

$$
A \cup B \prec_{2^{-k}}\left(\Lambda_{f(k)} \cup \Lambda_{g(k)}\right) \prec_{2^{-k}} S
$$

for each $k \in \mathbb{N}$. Let $h: \mathbb{N} \rightarrow \mathbb{N}$ be a computable function such that $[h(k)]=[f(k)] \cup[g(k)]$ for each $k \in \mathbb{N}$. Then $\Lambda_{f(k)} \cup \Lambda_{g(k)}=\alpha([f(k)] \cup[g(k)])=\Lambda_{h(k)}$ for each $k \in \mathbb{N}$ and the claim follows. 
Proposition 4.1 implies: if $A_{1}, \ldots, A_{n} \subseteq S$ and $A_{i}$ is computable up to $S$ for each $i \in\{1, \ldots, n\}$, then $A_{1} \cup \cdots \cup A_{n}$ is computable up to $S$. Therefore, we have the following statement.

Proposition 4.2. Let $(X, d, \alpha)$ be a computable metric space. Let $S$ be a compact subset of $(X, d)$ and suppose $A_{1}, \ldots, A_{n}$ are subsets of $S$ such that $S=A_{1} \cup \cdots \cup A_{n}$ and such that $A_{i}$ is computable up to $S$ for each $i \in\{1, \ldots, n\}$. Then $S$ is a computable compact set.

\section{LOCALIZATION OF COMPUTABILITY}

That $S$ is a semi-computable compact set in a computable metric space means that we can effectively enumerate all rational open sets which contain $S$. However we don't know, in general, how close $U$ is to $S$ for a given rational open set $U$ containing $S$ if $S$ is not computable. On the other hand, if we can, for a given $\varepsilon>0$, effectively find a rational open set $U$ which contains $S$ and such that $U \prec_{\varepsilon} S$, then we will have $S \approx_{\varepsilon} U$ and it will follow from Proposition 2.6 that $S$ is a computable compact set.

In order to effectively find, for a given $\varepsilon>0$, such a rational open set $U$, it would be enough to effectively find finitely many sets $C_{0}, \ldots, C_{m}$ whose union contains $S$, such that the diameter of each of these sets is less than $\varepsilon$ and such that each of these sets intersects $S$. Then $C_{0} \cup \cdots \cup C_{m} \prec_{\varepsilon} S$ and the computability of $S$ would follow.

This can be done in certain situations. For example, let us observe the case when $S$ is an arc. Then for each $\varepsilon>0$ there exists a finite sequence of rational open sets $C_{0}, \ldots, C_{m}$ whose union contains $S$ such that $C_{i} \cap C_{j}=\emptyset$ whenever $|i-j|>1$ and such that $\operatorname{diam}\left(C_{i}\right)<\varepsilon$ for each $i \in\{0, \ldots, m\}$. And if $S$ is a semi-computable compact set, a sequence $C_{0}, \ldots, C_{m}$ with these properties can be found effectively. Still, this is not enough to conclude that each of these sets intersects $S$. However, if the arc $S$ has computable endpoints $a$ and $b$, then the sequence $C_{0}, \ldots, C_{m}$ can be found so that $a \in C_{0}$ and $b \in C_{m}$. Now we can conclude that each $C_{i}$ intersects $S$, otherwise $C_{0} \cup \cdots \cup C_{i-1}$ and $C_{i+1} \cup \cdots \cup C_{m}$ would be disjoint open sets, each of them would intersect $S$ and $S$ would be contained in their union which is impossible since $S$ is connected.

Suppose now that $S$ is homeomorphic to $[0,1] \times[0,1]$. We can proceed in a similar way as in the case of an arc. For a given $\varepsilon>0$ we want sets $C_{i, j}, 0 \leq i, j \leq m$ whose union contains $S$, whose diameters are less then $\varepsilon$ and such that $C_{i, j}$ and $C_{i^{\prime}, j^{\prime}}$ are disjoint when $\left|i-i^{\prime}\right|>1$ or $\left|j-j^{\prime}\right|>1$. It turns out that, under some additional assumptions, we can do this in a satisfactory way [7], however when at the end we want to conclude that each of these sets intersects $S$, the fact that $S$ is connected is not enough for this conclusion and here we need some deeper topological facts about the space $[0,1]^{n}$ (see the discussion on pages 5,6 and 7 in [7]).

For $n \geq 1$ and $i \in\{1, \ldots, n\}$ let

$$
\begin{gathered}
A_{i}^{n}=\left\{\left(x_{1}, \ldots, x_{n}\right) \in[-2,2]^{n} \mid x_{i}=-2\right\}, \\
B_{i}^{n}=\left\{\left(x_{1}, \ldots, x_{n}\right) \in[-2,2]^{n} \mid x_{i}=2\right\} .
\end{gathered}
$$

(Here $[-2,2]^{n}$ denotes the set $\left\{\left(x_{1}, \ldots, x_{n}\right) \mid x_{1}, \ldots, x_{n} \in[-2,2]\right\}$.) When the context is clear, we write $A_{i}$ and $B_{i}$ instead of $A_{i}^{n}$ and $B_{i}^{n}$.

The following topological fact will be crucial in the proof of our main result (see [4, Theorem 1.8.1, and [7], Corollary 3.3.). 
Theorem 5.1. Let $n \geq 1$. Suppose $U_{1}, \ldots, U_{n}$ and $V_{1}, \ldots, V_{n}$ are open subsets of $\mathbb{R}^{n}$ such that

$$
U_{i} \cap A_{i}=\emptyset, V_{i} \cap B_{i}=\emptyset \text { and } U_{i} \cap V_{i}=\emptyset
$$

for all $i \in\{1, \ldots, n\}$. Then $[-2,2]^{n}$ is not contained in the union $U_{1} \cup \cdots \cup U_{n} \cup V_{1} \cup \cdots \cup V_{n}$.

Let $X$ be a set, $n \geq 1$ and $m \in \mathbb{N}$. A function

$$
C: \mathbb{N}_{m}^{n} \rightarrow \mathcal{P}(X)
$$

is called an $n$-chain in $X$ (of length $m$ ) if

$$
C_{i_{1}, \ldots, i_{n}} \cap C_{j_{1}, \ldots, j_{n}}=\emptyset
$$

for all $\left(i_{1}, \ldots, i_{n}\right),\left(j_{1}, \ldots, j_{n}\right) \in \mathbb{N}_{m}^{n}$ such that $\left|i_{l}-j_{l}\right|>1$ for some $l \in\{1, \ldots, n\}$. Here we use $C_{i_{1}, \ldots, i_{n}}$ to denote $C\left(i_{1}, \ldots, i_{n}\right)$.

For $a, b \in \mathbb{N}_{m}^{n}, a=\left(a_{1}, \ldots, a_{n}\right), b=\left(b_{1}, \ldots, b_{n}\right)$, we define the number $p(a, b)$ by

$$
p(a, b)=\max _{1 \leq i \leq n}\left|a_{i}-b_{i}\right| \text {. }
$$

With this notation, we have that $C: \mathbb{N}_{m}^{n} \rightarrow \mathcal{P}(X)$ is an $n$-chain if and only if $C_{a} \cap C_{b}=\emptyset$ for all $a, b \in \mathbb{N}_{m}^{n}$ such that $p(a, b)>1$. If $a \in \mathbb{N}_{m}^{n}$, we say that $C_{a}$ is a link of the chain $C$.

If $(X, d)$ is a metric space, then we say that an $n$-chain $C=\left(C_{i_{1}, \ldots, i_{n}}\right)_{0 \leq i_{1}, \ldots, i_{n} \leq m}$ in $X$ is open if $C_{i_{1}, \ldots, i_{n}}$ is an open set in $(X, d)$ for all $i_{1}, \ldots, i_{n} \in \mathbb{N}_{m}$. We similarly define the notion of a compact $n$-chain in $(X, d)$.

If $C: \mathbb{N}_{m}^{n} \rightarrow \mathcal{P}(X), C=\left(C_{i_{1}, \ldots, i_{n}}\right)_{0 \leq i_{1}, \ldots, i_{n} \leq m}$ is a function, then the function

$$
\left(C_{i_{1}, \ldots, i_{n-1}, 0}\right)_{0 \leq i_{1}, \ldots, i_{n-1} \leq m}
$$

is called the lower boundary of $C$. If $C$ is an $n$-chain in $(X, d)$, then its lower boundary is an $n$-1-chain in $(X, d)$.

In general, if $A$ is a set and $f: A \rightarrow \mathcal{P}(X)$ a function, we will denote by $\bigcup f$ the union $\bigcup_{a \in A} f(a)$ and we will say that $f$ covers $S$, where $S \subseteq X$, if $S \subseteq \bigcup f$. If $(X, d)$ is a metric space, $A$ a nonempty finite set and $f(a)$ a nonempty bounded set for each $a \in A$, then we define $\operatorname{mesh}(f)$ as the number

$$
\operatorname{mesh}(f)=\max _{a \in A}(\operatorname{diam} f(a)) .
$$

Let $\varepsilon>0$. An $n$-chain $C$ in a metric space $(X, d)$ is said to be an $\varepsilon-n$-chain if $\operatorname{mesh}(C)<\varepsilon$.

Let $n \geq 1$. A finite $n$-sequence in $\mathbb{N}$ is any function of the form

$$
\{0, \ldots, m\}^{n} \rightarrow \mathbb{N}
$$

Recall that any finite sequence $i_{0}, \ldots, i_{m}$ in $\mathbb{N}$ is of the form $(j)_{0}, \ldots,(j)_{\bar{j}}$ for some $j \in \mathbb{N}$. Let $\nu: \mathbb{N}^{n} \rightarrow \mathbb{N}$ be some fixed computable injection and let $\tau_{1}$ and $\tau_{2}$ be the functions from the section 2 . We define $\Sigma: \mathbb{N}^{n+1} \rightarrow \mathbb{N}$ by

$$
\Sigma\left(i, j_{1}, \ldots, j_{n}\right)=\left(\tau_{1}(i)\right)_{\nu\left(j_{1}, \ldots, j_{n}\right)}
$$

Then for any finite $n$-sequence $a$ in $\mathbb{N}$ there exists $i \in \mathbb{N}$ such that $a$ equals the function

$$
\begin{gathered}
\left\{0, \ldots, \tau_{2}(i)\right\}^{n} \rightarrow \mathbb{N}, \\
\left(j_{1}, \ldots, j_{n}\right) \mapsto \Sigma\left(i, j_{1}, \ldots, j_{n}\right) .
\end{gathered}
$$


We will use the following notation: $\widehat{i}$ instead of $\tau_{2}(i)$ and, for $n \geq 2,(i)_{j_{1}, \ldots, j_{n}}$ instead of $\Sigma\left(i, j_{1}, \ldots, j_{n}\right)$.

Let $(X, d, \alpha)$ be a computable metric space. For $l \in \mathbb{N}$ let $\mathcal{H}_{l}$ be the finite $n$-sequence of sets in $X$ defined by

$$
\mathcal{H}_{l}=\left(J_{(l)_{j_{1}, \ldots, j_{n}}}\right)_{0 \leq j_{1}, \ldots, j_{n} \leq \widehat{l}}
$$

(i.e. $\mathcal{H}_{l}$ is the function $\{0, \ldots, \widehat{l}\}^{n} \rightarrow \mathcal{P}(X)$ which maps $\left(j_{1}, \ldots, j_{n}\right)$ to $\left.J_{(l)_{j_{1}, \ldots, j_{n}}}\right)$.

Lemma 5.2. Let $(X, d, \alpha)$ be a computable metric space. Then there exist computable functions $\zeta, \zeta^{\prime}: \mathbb{N} \rightarrow \mathbb{N}$ such that, for each $l \in \mathbb{N}$, the set $J_{\zeta(l)}$ is the union of $\mathcal{H}_{l}$ and $J_{\zeta^{\prime}(l)}$ is the union of the lower boundary of $\mathcal{H}_{l}$.

Proof. See Lemma 4.3. in [7].

Proposition 5.3. Let $(X, d, \alpha)$ be a computable metric space and let $S$ be a semi-computable compact set in this space.

(i) The set $\left\{l \in \mathbb{N} \mid \mathcal{H}_{l}\right.$ covers $\left.S\right\}$ is c.e.

(ii) The set $\left\{l \in \mathbb{N} \mid\right.$ the lower boundary of $\mathcal{H}_{l}$ covers $\left.S\right\}$ is c.e.

Proof. Let $\zeta, \zeta$ be the functions from Lemma 5.2. Then $\mathcal{H}_{l}$ covers $S$ if and only if $S \subseteq J_{\zeta(l)}$ and the lower boundary of $\mathcal{H}_{l}$ covers $S$ if and only $S \subseteq J_{\zeta^{\prime}(l)}$. Claims (i) and (ii) follow.

We say that $\mathcal{H}_{l}$ is a formal $n$-chain if $J_{(l)_{a}}$ and $J_{(l)_{b}}$ are formally disjoint for all $a, b \in \mathbb{N}_{\widehat{l}}^{n}$ such that $p(a, b)>1$.

Let the function fmesh $: \mathbb{N} \rightarrow \mathbb{R}$ be defined by

$$
\operatorname{fmesh}(l)=\max _{0 \leq j_{1}, \ldots, j_{n} \leq \hat{l}} \operatorname{fdiam}\left((l)_{j_{1}, \ldots, j_{n}}\right) .
$$

\section{Proposition 5.4.}

(i) The function fmesh is computable.

(ii) The set $\left\{l \in \mathbb{N} \mid \mathcal{H}_{l}\right.$ is a formal n-chain $\}$ is computably enumerable.

Proof. (i) follows from Proposition 2.4(1) and Proposition 2.2(4).

Let us prove (ii). Let $\Phi: \mathbb{N} \rightarrow \mathcal{P}\left(\mathbb{N}^{2 n+1}\right)$ be defined by $\Phi(l)=\left\{\left(l, v_{1}, \ldots, v_{n}, w_{1}, \ldots\right.\right.$ $\left.\ldots, w_{n}\right)\left|0 \leq v_{1}, \ldots, v_{n}, w_{1}, \ldots, w_{n} \leq \widetilde{l},\right| v_{i}-w_{i} \mid>1$ for some $\left.i \in\{1, \ldots, n\}\right\}$. Then $\Phi$ is c.c.b. Let $\Psi: \mathbb{N}^{2 n+1} \rightarrow \mathcal{P}\left(\mathbb{N}^{2}\right)$ be defined by

$$
\Psi\left(l, v_{1}, \ldots, v_{n}, w_{1}, \ldots, w_{n}\right)=\left\{\left((l)_{v_{1}, \ldots, v_{n}},(l)_{w_{1}, \ldots, w_{n}}\right)\right\} .
$$

Clearly, $\Psi$ is c.c.b. The set $\Omega$ of all $(i, j) \in \mathbb{N}^{2}$ such that $J_{i}$ and $J_{j}$ are formally disjoint is c.e. by Proposition 2.4(2). Finally, the function $\Lambda: \mathbb{N} \rightarrow \mathcal{P}\left(\mathbb{N}^{2}\right)$ defined by $\Phi$ and $\Psi$ as in Proposition 2.1(3) is c.c.b. and we have that $\mathcal{H}_{l}$ is a formal $n$-chain if and only if $\Lambda(l) \subseteq \Omega$. It follows now from Proposition 2.1 (4) that the set $\left\{l \in \mathbb{N} \mid \mathcal{H}_{l}\right.$ is a formal $n$-chain $\}$ is c.e.

If $(X, d)$ is a metric space, then for nonempty subsets $S$ and $T$ of $X$ we denote the number $\inf \{d(x, y) \mid x \in S, y \in T\}$ by $d(S, T)$. The proof of the following lemma is straightforward.

Lemma 5.5. Let $(X, d)$ be a metric space and let $K$ and $L$ be nonempty compact sets in this space. Let $r=\frac{d(K, L)}{4}$. Then, if $x, y \in X$ and $s, t \in \mathbb{R}$ are such that $0<s, t<r$ and $B(x, s) \cap K \neq \emptyset$ and $B(y, t) \cap L \neq \emptyset$, then $d(x, y)>s+t$. 
Let us suppose that $S$ is a semi-computable compact set in a computable metric space $(X, d, \alpha)$. Suppose $S$ is homeomorphic to $[0,1] \times[0,1]$. To show that $S$ is a computable compact set, it would be enough to effectively find, for a given $\varepsilon>0$, an $\varepsilon-2$-chain which covers $S$ and such that each of its links intersects $S$. The question whether a chain covers $S$ or not is semi-decidable since $S$ is a semi-computable compact set. But the problem is how to ensure that each link of a chain intersects $S$. In fact, this is not possible in general since $S$ needs not be computable. The idea we used to tackle this problem is to find some effective procedure which will, for a given $\varepsilon>0$, give an $\varepsilon-2$-chain which covers $S$ in such a way that we can be sure that certain links (which we can effectively determine) do intersect $S$. This idea is used in the proof of the following theorem.

Theorem 5.6. Let $(X, d, \alpha)$ be a computable metric space. Let $S$ be a semi-computable compact set in this space and suppose $x \in S$ is a point which has a neighborhood in $S$ homeomorphic to $\mathbb{R}^{n}$ for some $n \in \mathbb{N}$. Then there exists a neighborhood of $x$ in $S$ which is computable up to $S$.

Proof. Let $U$ be a neighborhood of $x$ in $S$ (i.e. $x \in \operatorname{Int}_{S} U$, where $\operatorname{Int}_{S} U$ is the interior of $U$ in $S$ ) and $n \in \mathbb{N}$ so that $U$ is homeomorphic to $\mathbb{R}^{n}$. We may assume that $U$ is open in $S$ (certainly $\operatorname{Int}_{S} U$ is homeomorphic to an open subset of $\mathbb{R}^{n}$ which implies that some open subset of $\operatorname{Int}_{S} U$ which contains $x$ is homeomorphic to an open ball in $\mathbb{R}^{n}$; however every open ball in $\mathbb{R}^{n}$ is homeomorphic to $\mathbb{R}^{n}$ ). Let $f: \mathbb{R}^{n} \rightarrow U$ be a homeomorphism. We may assume $f(0)=x$ (otherwise we take the composition of $f$ and some translation $\mathbb{R}^{n} \rightarrow \mathbb{R}^{n}$ ). The set $f\left(\langle-4,4\rangle^{n}\right)$ is open in $U$ and therefore in $S$ which implies that $S \backslash f\left(\langle-4,4\rangle^{n}\right)$ is closed in $S$. (Here, for $a, b \in \mathbb{R}$, we use $\langle a, b\rangle$ to denote the open interval $\{x \in \mathbb{R} \mid a<x<b\}$.) Hence $S \backslash f\left(\langle-4,4\rangle^{n}\right)$ is compact and since it is disjoint with $f\left([-2,2]^{n}\right)$, which is clearly compact, there exists $m_{0} \in \mathbb{N}$ such that the rational open set $J_{m_{0}}$ satisfies the following:

$$
S \backslash f\left(\langle-4,4\rangle^{n}\right) \subseteq J_{m_{0}} \text { and } J_{m_{0}} \cap f\left([-2,2]^{n}\right)=\emptyset .
$$

Let $S^{\prime}=S \backslash J_{m_{0}}$. Then, by Lemma $3.3, S^{\prime}$ is a semi-computable compact set in $(X, d, \alpha)$ and

$$
f\left([-2,2]^{n}\right) \subseteq S^{\prime} \subseteq f\left([-4,4]^{n}\right)
$$

See Figure 1. The areas bounded by the inside, the middle and the outside curve are $f\left([-1,1]^{n}\right), f\left([-2,2]^{n}\right)$ and $f\left([-4,4]^{n}\right)$ respectively. The grey area is $S^{\prime}$.

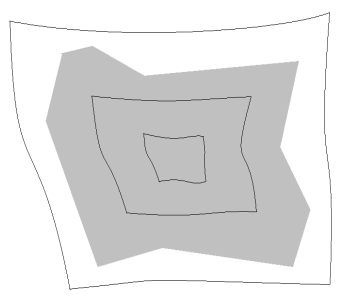

Figure 1.

For $i \in\{1, \ldots, n\}$ let

$$
C_{i}=\left\{\left(x_{1}, \ldots, x_{n}\right) \in[-4,4]^{n} \mid x_{i} \geq-1\right\}, D_{i}=\left\{\left(x_{1}, \ldots, x_{n}\right) \in[-4,4]^{n} \mid x_{i} \leq 1\right\} .
$$

Note that for each $i \in\{1, \ldots, n\}$ the sets $f\left(A_{i}\right)$ and $f\left(C_{i}\right)$ are compact and disjoint and the sets $f\left(B_{i}\right)$ and $f\left(D_{i}\right)$ are compact and disjoint (recall that $A_{i}$ and $B_{i}$ are defined by (5.1) 
and $(5.2)$ ). On Figure 2 the blue area is $f\left(A_{i}\right)$ and the green area is $f\left(C_{i}\right)$. On Figure 3 the blue area is $f\left(B_{i}\right)$ and the green area is $f\left(D_{i}\right)$.

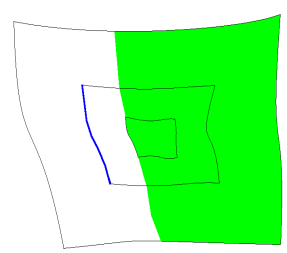

Figure 2.

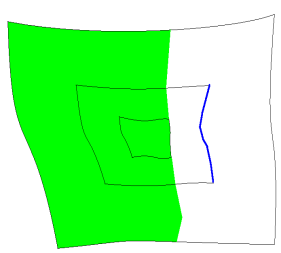

Figure 3.

Also, $\{x\}$ and $f\left([-4,4]^{n} \backslash\langle-1,1\rangle^{n}\right)$ are compact and disjoint. Choose a positive rational number $\gamma$ such that

$$
\gamma<\frac{\left.d\left(f\left(A_{i}\right)\right), f\left(C_{i}\right)\right)}{4}, \gamma<\frac{\left.d\left(f\left(B_{i}\right)\right), f\left(D_{i}\right)\right)}{4} \text { and } \gamma<\frac{d\left(\{x\}, f\left([-4,4]^{n} \backslash\langle-1,1\rangle^{n}\right)\right)}{4}
$$

for each $i \in\{1, \ldots, n\}$.

Let $i \in\{1, \ldots, n\}$. Let $\mathcal{U}=\left\{B\left(\alpha_{l}, \gamma\right) \mid l \in \mathbb{N}\right\}$. Then $\mathcal{U}$ is an open cover for $f\left(A_{i}\right)$ in $(X, d)$ and therefore there exists a finite subcover $\mathcal{U}^{\prime}$ of $\mathcal{U}$ for $f\left(A_{i}\right)$. Let $\mathcal{U}^{\prime \prime}$ be the subset of $\mathcal{U}^{\prime}$ consisting of those members of $\mathcal{U}^{\prime}$ which intersect $f\left(A_{i}\right)$. We have

$$
\mathcal{U}^{\prime \prime}=\left\{B\left(\alpha_{l_{0}}, \gamma\right), \ldots, B\left(\alpha_{l_{k}}, \gamma\right)\right\}
$$

where $l_{0}, \ldots, l_{k} \in \mathbb{N}$. Choose $\omega \in \mathbb{N}$ such that $\gamma=q_{\omega}$. Let $v_{0}, \ldots, v_{k} \in \mathbb{N}$ be such that $\tau_{1}\left(v_{j}\right)=l_{j}$ and $\tau_{2}\left(v_{j}\right)=\omega$ for each $j \in\{0, \ldots, k\}$. Finally, choose $a_{i} \in \mathbb{N}$ so that

$$
\left[a_{i}\right]=\left\{v_{0}, \ldots, v_{k}\right\} \text {. }
$$

In this way we have defined the numbers $a_{1}, \ldots, a_{n}$. For each $i \in\{1, \ldots, n\}$ clearly $f\left(A_{i}\right) \subseteq$ $J_{a_{i}}$ and if $j \in \mathbb{N}$ is such that $I_{j} \cap f\left(C_{i}\right) \neq \emptyset$ and $\rho_{j}<\gamma$, then by (5.5) and Lemma 5.5 we have that $I_{j}$ and $J_{a_{i}}$ are formally disjoint.

In the same way we define numbers $b_{1}, \ldots, b_{n}$ with the following property: if $i \in$ $\{1, \ldots, n\}$, then $f\left(B_{i}\right) \subseteq J_{b_{i}}$ and if $j \in \mathbb{N}$ is such that $I_{j} \cap f\left(D_{i}\right) \neq \emptyset$ and $\rho_{j}<\gamma$, then $I_{j}$ and $J_{b_{i}}$ are formally disjoint.

Similarly, there exists $x^{\prime} \in \mathbb{N}$ with the property that $x \in J_{x^{\prime}}$ and if $j \in \mathbb{N}$ is such that $I_{j} \cap f\left([-4,4]^{n} \backslash\langle-1,1\rangle^{n}\right) \neq \emptyset$ and $\rho_{j}<\gamma$, then $I_{j}$ and $J_{x^{\prime}}$ are formally disjoint.

For $m \in \mathbb{N}$ let $E^{m}: \mathbb{N}_{8 m+7}^{n} \rightarrow \mathcal{P}\left([-4,4]^{n}\right)$ be defined by

$$
E_{i_{1}, \ldots, i_{n}}^{m}=\left[-4+\frac{i_{1}}{m+1},-4+\frac{i_{1}+1}{m+1}\right] \times \cdots \times\left[-4+\frac{i_{n}}{m+1},-4+\frac{i_{n}+1}{m+1}\right] .
$$

Then $E^{m}$ is a compact $n$-chain in $[-4,4]^{n}$ which covers $[-4,4]^{n}$. Let $F^{m}: \mathbb{N}_{8 m+7}^{n} \rightarrow \mathcal{P}(X)$ be defined by $F^{m}(v)=f\left(E^{m}(v)\right), v \in \mathbb{N}_{8 m+7}^{n}$. Then $F^{m}$ is a compact $n$-chain in $(X, d)$ which covers $f\left([-4,4]^{n}\right)$.

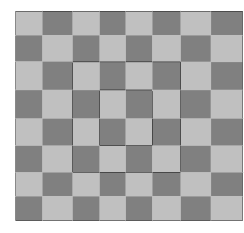

Figure 4. Chain $E^{m}$

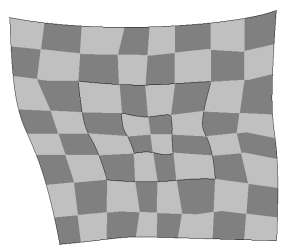

Figure 5. Chain $F^{m}$ 
Let $\varepsilon>0$. Since the restriction of $f$ to $[-4,4]^{n}$ is uniformly continuous, there exists $m \in \mathbb{N}$ such that mesh $\left(F^{m}\right)<\frac{\varepsilon}{6}$. Let $\delta$ be a positive rational number such that $\delta<\gamma$, $\delta<\frac{\varepsilon}{6}$ and

$$
\delta<\frac{d\left(F^{m}(v), F^{m}(w)\right)}{4}
$$

for all $v, w \in \mathbb{N}_{8 m+7}^{n}$ such that $p(v, w)>1$.

Now we define numbers $j_{v}$ for $v \in \mathbb{N}_{8 m+7}$ in the following way. Let $v \in \mathbb{N}_{8 m+7}^{n}$. As before, we conclude that there exist finitely many numbers $i_{0}, \ldots, i_{k}$ such that $F^{m}(v)$ is contained in the union of the balls $B\left(\alpha_{i_{0}}, \delta\right), \ldots, B\left(\alpha_{i_{k}}, \delta\right)$ and each of these balls intersects $F^{m}(v)$. Let $p \in \mathbb{N}$ be such that $q_{p}=\delta$. Now, let $w_{0}, \ldots, w_{k}$ be such that $\tau_{1}\left(w_{l}\right)=i_{l}$, $\tau_{2}\left(w_{l}\right)=p$ for each $l \in\{0, \ldots, k\}$. Finally, choose $j_{v} \in \mathbb{N}$ so that $\left[j_{v}\right]=\left\{w_{0}, \ldots, w_{m}\right\}$.

We conclude readily that fdiam $\left(j_{v}\right)<\varepsilon$ for each $v \in \mathbb{N}_{8 m+7}^{n}$ and it also follows, using Lemma 5.5, that $J_{j_{v}}$ and $J_{j_{w}}$ are formally disjoint for all $v, w \in \mathbb{N}_{8 m+7}^{n}$ such that $p(v, w)>1$. Furthermore, let $v \in \mathbb{N}_{8 m+7}^{n}, v=\left(v_{1}, \ldots, v_{n}\right)$ and let $i \in\{1, \ldots, n\}$. If $v_{i} \geq 3 m+3$, then $F^{m}(v) \subseteq f\left(C_{i}\right)$ and by the construction of $j_{v}$ and $a_{i}$ we have that $J_{j_{v}}$ and $J_{a_{i}}$ are formally disjoint. Similarly, if $v_{i} \leq 5 m+4$, then $F^{m}(v) \subseteq f\left(D_{i}\right)$ and we get that $J_{j_{v}}$ and $J_{b_{i}}$ are formally disjoint. Also note that $v_{i}<3 m+3$ or $v_{i}>5 m+4$ implies that $J_{j_{v}}$ and $J_{x^{\prime}}$ are formally disjoint. Note that $S^{\prime}$ (in fact $f\left([-4,4]^{n}\right.$ ) is contained in the union of the sets $J_{j_{v}}$, $v \in \mathbb{N}_{8 m+7}^{n}$.

Choose $l \in \mathbb{N}$ such that $\widehat{l}=8 m+7$ and such that $j_{v}=(l)_{v}$ for each $v \in \mathbb{N}_{8 m+7}^{n}$. Put $e=3 m+3, h=5 m+4$. The preceding construction can be made for $\varepsilon=2^{-k}$ for each $k \in \mathbb{N}$. We have the following conclusions:

(1) $\mathcal{H}_{l}$ covers $S^{\prime}$

(2) $\mathcal{H}_{l}$ is a formal $n$-chain;

(3) $J_{(l)_{v_{1}, \ldots, v_{n}}}$ and $J_{a_{i}}$ are formally disjoint if $1 \leq i \leq n, 0 \leq v_{1}, \ldots, v_{n} \leq \widehat{l}$ and $v_{i} \geq e$;

(4) $J_{(l)_{v_{1}, \ldots, v_{n}}}$ and $J_{b_{i}}$ are formally disjoint if $1 \leq i \leq n, 0 \leq v_{1}, \ldots, v_{n} \leq \widehat{l}$ and $v_{i} \leq h$;

(5) $J_{(l)_{v_{1}, \ldots, v_{n}}}$ and $J_{x^{\prime}}$ are formally disjoint if $1 \leq i \leq n, 0 \leq v_{1}, \ldots, v_{n} \leq \widehat{l}$ and $\left(v_{i}<e\right.$ or $\left.v_{i}>h\right)$

(6) $\operatorname{fmesh}(l)<2^{-k}$.

These conditions are semi-decidable, i.e. the set $\Omega$ of all $(k, l, e, h) \in \mathbb{N}^{4}$ such that (1)-(6) hold is c.e. (Proposition 5.3, Proposition 5.4 and Proposition 2.2(3) imply that conditions (1), (2) and (6) are semi-decidable; that (3)-(5) are semi-decidable can be seen as in the proof of Proposition 5.4). Since for each $k \in \mathbb{N}$ there exist $l, e, h \in \mathbb{N}$ such that $(k, l, e, h) \in \Omega$, there exist computable functions $\tilde{l}, \tilde{e}, \tilde{h}: \mathbb{N} \rightarrow \mathbb{N}$ such that $(k, \tilde{l}(k), \tilde{e}(k), \tilde{h}(k)) \in \Omega$ for each $k \in \mathbb{N}$. Let $k \in \mathbb{N}$ and let $l=\tilde{l}(k), e=\tilde{e}(k)$ and $h=\tilde{h}(k)$. Let $\Gamma$ be the set of all $\left(v_{1}, \ldots, v_{n}\right) \in \mathbb{N}_{\widehat{l}}^{n}$ such that $e \leq v_{i} \leq h$ for each $i \in\{1, \ldots, n\}$. We claim that

$$
J_{(l)_{v}} \cap f\left([-2,2]^{n}\right) \neq \emptyset \text { for all } v \in \Gamma,
$$

and

$$
S^{\prime} \cap J_{x^{\prime}} \subseteq \bigcup_{v \in \Gamma} J_{(l)_{v}} .
$$

Suppose $J_{(l)_{v}} \cap f\left([-2,2]^{n}\right)=\emptyset$ for some $v=\left(v_{1}, \ldots, v_{n}\right) \in \Gamma$. 

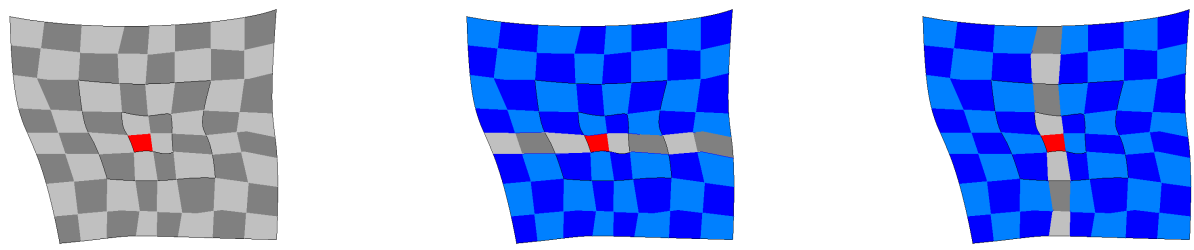

Figure 6. The red link is $J_{(l)_{v}}$.

For each $i \in\{1, \ldots, n\}$ we define $U_{i}$ as the union of all $J_{(l)_{w_{1}, \ldots, w_{n}}}$ such that $\left(w_{1}, \ldots, w_{n}\right) \in$ $\mathbb{N}_{\widehat{l}}^{n}$ and $w_{i}<v_{i}$ and we define $V_{i}$ as the union of all $J_{(l)_{w_{1}, \ldots, w_{n}}}$ such that $\left(w_{1}, \ldots, w_{n}\right) \in \mathbb{N}_{\widehat{l}}^{n}$ and $w_{i}>v_{i}$. (In Figure 6 the sets $U_{i}$ are represented by the bottom and the left blue set and the sets $V_{i}$ by the upper and the right blue set.) Then

$$
f\left([-2,2]^{n}\right) \subseteq\left(U_{1} \cup \cdots \cup U_{n}\right) \cup\left(V_{1} \cup \cdots \cup V_{n}\right) .
$$

Since $\mathcal{H}_{l}$ is a chain, $U_{i} \cap V_{i}=\emptyset$ for each $i \in\{1, \ldots, n\}$. Since $v_{i} \leq h$, by property (4) we have $U_{i} \cap J_{b_{i}}=\emptyset$, hence $U_{i} \cap f\left(B_{i}\right)=\emptyset$. Similarly, $v_{i} \geq e$ implies $V_{i} \cap J_{a_{i}}=\emptyset$ and this gives $V_{i} \cap f\left(A_{i}\right)=\emptyset$. Therefore

$$
f^{-1}\left(U_{i}\right) \cap B_{i}=\emptyset, f^{-1}\left(V_{i}\right) \cap A_{i}=\emptyset \text { and } f^{-1}\left(U_{i}\right) \cap f^{-1}\left(V_{i}\right)=\emptyset
$$

for each $i \in\{1, \ldots, n\}$ and

$$
[-2,2]^{n} \subseteq f^{-1}\left(U_{1}\right) \cup \cdots \cup f^{-1}\left(U_{n}\right) \cup f^{-1}\left(V_{1}\right) \cup \cdots \cup f^{-1}\left(V_{n}\right)
$$

This is impossible by Theorem 5.1. So (5.6) holds.

That (5.7) holds, it follows readily from property (5). Note that (5.7) implies $\Gamma \neq \emptyset$. Hence $\tilde{e}(k) \leq \tilde{h}(k)$ for each $k \in \mathbb{N}$.

Let $g: \mathbb{N} \rightarrow \mathbb{N}$ be some computable function such that

$$
\Lambda_{g(k)} \approx_{2^{-k}} \bigcup_{\tilde{e}(k) \leq v_{1}, \ldots, v_{n} \leq \tilde{h}(k)} J_{(\tilde{l}(k))_{v_{1}, \ldots, v_{n}}} .
$$

Such a function can be obtained by defining $\Phi: \mathbb{N} \rightarrow \mathcal{P}(\mathbb{N}), \Phi(k)=\left\{\tau_{1}\left(\left((l)_{v_{1}, \ldots, v_{n}}\right)_{0}\right) \mid\right.$ $\left.\tilde{e}(k) \leq v_{1}, \ldots, v_{n} \leq \tilde{h}(k)\right\}$ and taking a computable $g: \mathbb{N} \rightarrow \mathbb{N}$ such that $\Phi(k)=[g(k)]$ for each $k \in \mathbb{N}$.

By (5.6) and (5.7)

$$
S^{\prime} \cap J_{x^{\prime}} \prec_{2^{-k}} \Lambda_{g(k)} \text { and } \Lambda_{g(k)} \prec_{2 \cdot 2^{-k}} S
$$

for each $k \in \mathbb{N}$. Since $f\left(\langle-1,1\rangle^{n}\right)$ is an open set in $U$ and $U$ is open in $S, f\left(\langle-1,1\rangle^{n}\right)$ is open in $S$ and by (5.4) we have $f\left(\langle-1,1\rangle^{n}\right) \subseteq S^{\prime}$. Hence $S^{\prime}$ is a neighborhood of $x$ in $S$ and therefore $S^{\prime} \cap J_{x^{\prime}}$ is a neighborhood of $x$ in $S$. By (5.8) $S^{\prime} \cap J_{x^{\prime}}$ is computable up to $S$.

For $n \in \mathbb{N}, n \geq 1$, let

$$
\mathbb{H}^{n}=\left\{\left(x_{1}, \ldots, x_{n}\right) \in \mathbb{R}^{n} \mid x_{n} \geq 0\right\} .
$$

The subset of $\mathbb{H}^{n}$ consisting of those points whose last coordinate is zero is denoted by $\mathrm{Bd} \mathbb{H}^{n}$.

Theorem 5.7. Let $(X, d, \alpha)$ be a computable metric space and let $S$ and $T$ be semicomputable compact sets in this space such that $T \subseteq S$. Suppose $x \in S$ is a point which has a neighborhood $U$ (in $S$ ) with the following property: there exists a homeomorphism 
$f: \mathbb{H}^{n} \rightarrow U$ such that $f\left(\mathrm{Bd} \mathbb{H}^{n}\right)=U \cap T$. Then there exists a neighborhood of $x$ in $S$ which is computable up to $S$.

Proof. If $f^{-1}(x) \in \mathbb{H}^{n} \backslash\left(\mathrm{Bd} \mathbb{H}^{n}\right)$, then $x$ has a neighborhood homeomorphic to some open ball in $\mathbb{R}^{n}$, hence homeomorphic to $\mathbb{R}^{n}$ and the claim of the theorem follows from Theorem 5.6.

Suppose now that $f^{-1}(x) \in \mathrm{Bd} \mathbb{H}^{n}$. As in the proof of Theorem 5.6, we may assume that $U$ is open is $S$ and $f(0)=x$. As before we conclude that there exists $m_{0} \in \mathbb{N}$ such that

$$
S \backslash f\left(\langle-4,4\rangle^{n} \cap \mathbb{H}^{n}\right) \subseteq J_{m_{0}} \text { and } J_{m_{0}} \cap f\left([-2,2]^{n} \cap \mathbb{H}^{n}\right)=\emptyset .
$$

Let $S^{\prime}=S \backslash J_{m_{0}}$. By Lemma $3.3 S^{\prime}$ is a semi-computable compact set in $(X, d, \alpha)$ and

$$
f\left([-2,2]^{n} \cap \mathbb{H}^{n}\right) \subseteq S^{\prime} \subseteq f\left([-4,4]^{n} \cap \mathbb{H}^{n}\right) .
$$

Let $T^{\prime}=T \backslash J_{m_{0}}$. Then $T^{\prime}$ is semi-computable and

$$
f\left([-2,2]^{n} \cap\left(\mathrm{Bd} \mathbb{H}^{n}\right)\right) \subseteq T^{\prime} \subseteq f\left([-4,4]^{n} \cap\left(\mathrm{Bd} \mathbb{H}^{n}\right)\right) .
$$

For $i \in\{1, \ldots, n-1\}$ let

$C_{i}=\left\{\left(x_{1}, \ldots, x_{n}\right) \in[-4,4]^{n} \cap \mathbb{H}^{n} \mid x_{i} \geq-1\right\}, D_{i}=\left\{\left(x_{1}, \ldots, x_{n}\right) \in[-4,4]^{n} \cap \mathbb{H}^{n} \mid x_{i} \leq 1\right\}$.

Let

$$
D_{n}=\left\{\left(x_{1}, \ldots, x_{n}\right) \in[-4,4]^{n} \cap \mathbb{H}^{n} \mid x_{n} \leq 1\right\} .
$$

For $i \in\{1, \ldots, n-1\}$ let

$$
\begin{gathered}
\tilde{A}_{i}=\left\{\left(x_{1}, \ldots, x_{n}\right) \in[-2,2]^{n} \cap \mathbb{H}^{n} \mid x_{i}=-2\right\}, \\
\tilde{B}_{i}=\left\{\left(x_{1}, \ldots, x_{n}\right) \in[-2,2]^{n} \cap \mathbb{H}^{n} \mid x_{i}=2\right\} .
\end{gathered}
$$

Let

$\tilde{A}_{n}=\left\{\left(x_{1}, \ldots, x_{n}\right) \in[-2,2]^{n} \cap \mathbb{H}^{n} \mid x_{n}=0\right\}, \tilde{B}_{n}=\left\{\left(x_{1}, \ldots, x_{n}\right) \in[-2,2]^{n} \cap \mathbb{H}^{n} \mid x_{n}=2\right\}$.

For $m \in \mathbb{N}$ let $E^{m}: \mathbb{N}_{8 m+7}^{n} \rightarrow \mathcal{P}\left([-4,4]^{n} \cap \mathbb{H}^{n}\right)$ be defined so that $E_{i_{1}, \ldots, i_{n}}^{m}$ is equal to

$$
\left[-4+\frac{i_{1}}{m+1},-4+\frac{i_{1}+1}{m+1}\right] \times \cdots \times\left[-4+\frac{i_{n-1}}{m+1},-4+\frac{i_{n-1}+1}{m+1}\right] \times\left[\frac{i_{n}}{2 m+2}, \frac{i_{n}+1}{2 m+2}\right] .
$$

Then $E^{m}$ is a compact $n$-chain in $[-4,4]^{n} \cap \mathbb{H}^{n}$ which covers $[-4,4]^{n} \cap \mathbb{H}^{n}$. Let $F^{m}$ : $\mathbb{N}_{8 m+7}^{n} \rightarrow \mathcal{P}(X)$ be defined by $F^{m}(v)=f\left(E^{m}(v)\right), v \in \mathbb{N}_{8 m+7}^{n}$. Then $F^{m}$ is a compact $n$-chain in $(X, d)$ which covers $f\left([-4,4]^{n} \cap \mathbb{H}^{n}\right)$. Note that the lower boundary of $F^{m}$ covers $f\left([-4,4]^{n} \cap\left(\mathrm{Bd} \mathbb{H}^{n}\right)\right)$. Therefore by $(5.10)$ the lower boundary of $F^{m}$ covers $T^{\prime}$.

Exactly as in the proof of Theorem 5.6 we get numbers $a_{1}, \ldots, a_{n}, b_{1}, \ldots, b_{n}, x^{\prime} \in \mathbb{N}$ so that, for each $i \in\{1, \ldots, n\}, f\left(\tilde{A}_{i}\right) \subseteq J_{a_{i}}, f\left(\tilde{B}_{i}\right) \subseteq J_{b_{i}}, x \in J_{x^{\prime}}$ and so that for each $k \in \mathbb{N}$ there exist $l, e, h, u \in \mathbb{N}$ with the following properties:

(1) $\mathcal{H}_{l}$ covers $S^{\prime}$

(2) the lower boundary of $\mathcal{H}_{l}$ covers $T^{\prime}$;

(3) $\mathcal{H}_{l}$ is a formal $n$-chain;

(4) $J_{(l)_{v_{1}, \ldots, v_{n}}}$ and $J_{a_{i}}$ are formally disjoint if $1 \leq i \leq n-1,0 \leq v_{1}, \ldots, v_{n} \leq \widehat{l}$ and $v_{i} \geq e$;

(5) $J_{(l)_{v_{1}, \ldots, v_{n}}}$ and $J_{b_{i}}$ are formally disjoint if $1 \leq i \leq n-1,0 \leq v_{1}, \ldots, v_{n} \leq \widehat{l}$ and $v_{i} \leq h$;

(6) $J_{(l)_{v_{1}, \ldots, v_{n}}}$ and $J_{b_{n}}$ are formally disjoint if $0 \leq v_{1}, \ldots, v_{n} \leq \widehat{l}$ and $v_{n} \leq u$;

(7) $J_{(l)_{v_{1}, \ldots, v_{n}}}$ and $J_{x^{\prime}}$ are formally disjoint if $1 \leq i \leq n-1,0 \leq v_{1}, \ldots, v_{n} \leq \widehat{l}$ and $\left(v_{i}<e\right.$ or $\left.v_{i}>h\right)$ 
(8) $J_{(l)_{v_{1}, \ldots, v_{n}}}$ and $J_{x^{\prime}}$ are formally disjoint if $0 \leq v_{1}, \ldots, v_{n} \leq \widehat{l}$ and $v_{n}>u$;

(9) $\operatorname{fmesh}(l)<2^{-k}$.

The set $\Omega$ of all $(k, l, e, h, u) \in \mathbb{N}^{5}$ such that (1)-(9) hold is c.e. Therefore there exist computable functions $\tilde{l}, \tilde{e}, \tilde{h}, \tilde{u}: \mathbb{N} \rightarrow \mathbb{N}$ such that $(k, \tilde{l}(k), \tilde{e}(k), \tilde{h}(k), \tilde{u}(k)) \in \Omega$ for each $k \in \mathbb{N}$. Let $k \in \mathbb{N}$ and let $l=\tilde{l}(k), e=\tilde{e}(k), h=\tilde{h}(k)$ and $u=\tilde{u}(k)$. Let $\Gamma$ be the set of all $\left(v_{1}, \ldots, v_{n}\right) \in \mathbb{N}_{\widehat{l}}^{n}$ such that $v_{n} \leq u$ and $e \leq v_{i} \leq h$ for each $i \in\{1, \ldots, n-1\}$. We claim that

$$
J_{(l)_{v}} \cap f\left([-2,2]^{n} \cap \mathbb{H}^{n}\right) \neq \emptyset \text { for all } v \in \Gamma
$$

and

$$
S^{\prime} \cap J_{x^{\prime}} \subseteq \bigcup_{v \in \Gamma} J_{(l)_{v}}
$$

Suppose $J_{(l)_{v}} \cap f\left([-2,2]^{n}\right)=\emptyset$ for some $v=\left(v_{1}, \ldots, v_{n}\right) \in \Gamma$ such that $v_{n}=0$. For each $i \in\{1, \ldots, n-1\}$ let $U_{i}$ be the union of all $J_{(l)_{w_{1}, \ldots, w_{n}}}$ such that $\left(w_{1}, \ldots, w_{n}\right) \in \mathbb{N}_{l}^{n}, w_{n}=0$ and $w_{i}<v_{i}$ and let $V_{i}$ be the union of all $J_{(l)_{w_{1}, \ldots, w_{n}}}$ such that $\left(w_{1}, \ldots, w_{n}\right) \in \mathbb{N}_{\hat{l}}^{n}, w_{n}=0$ and $w_{i}>v_{i}$. It follows from (2) and (5.10) that

$$
f\left([-2,2]^{n} \cap\left(\mathrm{Bd} \mathbb{H}^{n}\right)\right) \subseteq\left(U_{1} \cup \cdots \cup U_{n-1}\right) \cup\left(V_{1} \cup \cdots \cup V_{n-1}\right) .
$$

Since $\mathcal{H}_{l}$ is a chain, $U_{i} \cap V_{i}=\emptyset$ for each $i \in\{1, \ldots, n-1\}$. Since $e \leq v_{i} \leq h$, by properties (4) and (5) we have $U_{i} \cap J_{b_{i}}=\emptyset$ and $V_{i} \cap J_{a_{i}}=\emptyset$. Hence $U_{i} \cap f\left(\tilde{B}_{i}\right)=\emptyset$ and $V_{i} \cap f\left(\tilde{A}_{i}\right)=\emptyset$. Therefore

$$
f^{-1}\left(U_{i}\right) \cap \tilde{B}_{i}=\emptyset, f^{-1}\left(V_{i}\right) \cap \tilde{A}_{i}=\emptyset \text { and } f^{-1}\left(U_{i}\right) \cap f^{-1}\left(V_{i}\right)=\emptyset
$$

for each $i \in\{1, \ldots, n-1\}$ and

$$
[-2,2]^{n} \cap\left(\mathrm{Bd} \mathbb{H}^{n}\right) \subseteq f^{-1}\left(U_{1}\right) \cup \cdots \cup f^{-1}\left(U_{n-1}\right) \cup f^{-1}\left(V_{1}\right) \cup \cdots \cup f^{-1}\left(V_{n-1}\right) .
$$

This is impossible by Theorem 5.1 (formally, we take the function $\gamma: \mathbb{R}^{n-1} \rightarrow \mathbb{H}^{n}, \gamma(z)=$ $(z, 0)$ and then for each $i \in\{1, \ldots, n-1\}$ we get from (5.13) that

$$
\gamma^{-1}\left(f^{-1}\left(U_{i}\right)\right) \cap B_{i}^{n-1}=\emptyset, \gamma^{-1}\left(f^{-1}\left(V_{i}\right)\right) \cap A_{i}^{n-1}=\emptyset, \gamma^{-1}\left(f^{-1}\left(U_{i}\right)\right) \cap \gamma^{-1}\left(f^{-1}\left(V_{i}\right)\right)=\emptyset
$$

which is impossible since the sets $\gamma^{-1}\left(f^{-1}\left(U_{1}\right)\right), \ldots, \gamma^{-1}\left(f^{-1}\left(U_{n-1}\right)\right), \gamma^{-1}\left(f^{-1}\left(V_{1}\right)\right), \ldots$, $\gamma^{-1}\left(f^{-1}\left(V_{n-1}\right)\right)$ are open in $\mathbb{R}^{n-1}$ and cover $[-2,2]^{n-1}=I^{n-1}$.)

So $J_{(l)_{v}} \cap f\left([-2,2]^{n}\right) \neq \emptyset$ for each $v=\left(v_{1}, \ldots, v_{n}\right) \in \Gamma$ such that $v_{n}=0$.

Let $v=\left(v_{1}, \ldots, v_{n}\right) \in \Gamma$ be such that $v_{n}>0$. Suppose $J_{(l)_{v}} \cap f\left([-2,2]^{n}\right)=\emptyset$.

For $i \in\{1, \ldots, n\}$ we define $U_{i}$ as the union of all $J_{(l)_{w_{1}, \ldots, w_{n}}}$ such that $\left(w_{1}, \ldots, w_{n}\right) \in \mathbb{N}_{\widehat{l}}^{n}$ and $w_{i}<v_{i}$ and we define $V_{i}$ as the union of all $J_{(l)_{w_{1}, \ldots, w_{n}}}$ such that $\left(w_{1}, \ldots, w_{n}\right) \in \mathbb{N}_{\hat{l}}^{n}$ and $w_{i}>v_{i}$. As before we conclude that

$$
f\left([-2,2]^{n} \cap \mathbb{H}^{n}\right) \subseteq\left(U_{1} \cup \cdots \cup U_{n-1}\right) \cup\left(V_{1} \cup \cdots \cup V_{n-1}\right)
$$

and

$$
U_{i} \cap V_{i}=\emptyset, U_{i} \cap f\left(\tilde{B}_{i}\right)=\emptyset, V_{i} \cap f\left(\tilde{A}_{i}\right)=\emptyset
$$

for each $i \in\{1, \ldots, n-1\}$. We also have $U_{n} \cap V_{n}=\emptyset$. Since $v_{n} \leq u$, property (6) implies $U_{n} \cap f\left(\tilde{B}_{n}\right)=\emptyset$. On the other hand, by 5.10 we have $f\left(\tilde{A}_{n}\right) \subseteq T^{\prime}$ and this, together with property (2), implies $f\left(\tilde{A}_{n}\right) \subseteq U_{n}$. Therefore $f\left(\tilde{A}_{n}\right) \cap V_{n}=\emptyset$.

We have the following conclusion: for each $i \in\{i, \ldots, n\}$

$$
f^{-1}\left(U_{i}\right) \cap \tilde{B}_{i}=\emptyset, f^{-1}\left(V_{i}\right) \cap \tilde{A}_{i}=\emptyset, f^{-1}\left(U_{i}\right) \cap f^{-1}\left(V_{i}\right)=\emptyset
$$


and

$$
[-2,2]^{n} \cap \mathbb{H}^{n} \subseteq f^{-1}\left(U_{1}\right) \cup \cdots \cup f^{-1}\left(U_{n}\right) \cup f^{-1}\left(V_{1}\right) \cup \cdots \cup f^{-1}\left(V_{n}\right) .
$$

To get a contradiction with Theorem 5.1 we define the function $\gamma: \mathbb{R}^{n} \rightarrow \mathbb{H}^{n}$ by

$$
\gamma\left(z_{1}, \ldots, z_{n}\right)=\left\{\begin{array}{l}
\left(z_{1}, \ldots, z_{n-1}, \frac{z_{n}+2}{2}\right), \text { if } z_{n} \geq-2 \\
\left(z_{1}, \ldots, z_{n-1}, 0\right), \text { if } z_{n} \leq-2
\end{array}\right.
$$

This is clearly a continuous function and we have $\gamma\left([-2,2]^{n}\right)=[-2,2]^{n} \cap \mathbb{H}^{n}$. It follows

$$
[-2,2]^{n} \subseteq \gamma^{-1}\left(f^{-1}\left(U_{1}\right)\right) \cup \cdots \cup \gamma^{-1}\left(f^{-1}\left(U_{n}\right)\right) \cup \gamma^{-1}\left(f^{-1}\left(V_{1}\right)\right) \cup \cdots \cup \gamma^{-1}\left(f^{-1}\left(V_{n}\right)\right) .
$$

For each $i \in\{1, \ldots, n\}$ clearly $\gamma^{-1}\left(f^{-1}\left(U_{i}\right)\right) \cap \gamma^{-1}\left(f^{-1}\left(V_{i}\right)\right)=\emptyset$,

$$
\gamma^{-1}\left(f^{-1}\left(U_{i}\right)\right) \cap \gamma^{-1}\left(\tilde{B}_{i}\right)=\emptyset \text { and } \gamma^{-1}\left(f^{-1}\left(V_{i}\right)\right) \cap \gamma^{-1}\left(\tilde{A}_{i}\right)=\emptyset .
$$

It is immediate from the definition of $\gamma$ that $A_{i}^{n} \subseteq \gamma^{-1}\left(\tilde{A}_{i}\right)$ and $B_{i}^{n} \subseteq \gamma^{-1}\left(\tilde{B}_{i}\right)$ for each $i \in\{1, \ldots, n\}$, therefore $\gamma^{-1}\left(f^{-1}\left(U_{i}\right)\right) \cap B_{i}^{n}=\emptyset$ and $\gamma^{-1}\left(f^{-1}\left(V_{i}\right)\right) \cap A_{i}^{n}=\emptyset$. This altogether contradicts Theorem 5.1. So we finally conclude that (5.11) holds.

That (5.12) holds, it follows from properties (7) and (8). We note as before that $\Gamma \neq \emptyset$ and therefore $\tilde{e}(k) \leq \tilde{h}(k)$ for each $k \in \mathbb{N}$.

Let $g: \mathbb{N} \rightarrow \mathbb{N}$ be some computable function such that

$$
\Lambda_{g(k)} \approx_{2^{-k}} \bigcup_{\tilde{e}(k) \leq v_{1}, \ldots, v_{n-1} \leq \tilde{h}(k), 0 \leq v_{n} \leq \tilde{u}(k)} J_{(\tilde{l}(k))_{v_{1}, \ldots, v_{n}}} .
$$

By (5.11) and (5.12)

$$
S^{\prime} \cap J_{x^{\prime}} \prec_{2^{-k}} \Lambda_{g(k)} \text { and } \Lambda_{g(k)} \prec_{2 \cdot 2^{-k}} S
$$

for each $k \in \mathbb{N}$. Since $S^{\prime} \cap J_{x^{\prime}}$ is a neighborhood of $x$ in $S$, the claim of the theorem follows.

\section{SEMI-COMPUTABLE COMPACT MANIFOLDS With BOUNDARY}

Let $n \in \mathbb{N}, n \geq 1$. A nonempty topological space $X$ is said to be an $n$-manifold if each point of $X$ has a neighborhood which is homeomorphic to $\mathbb{R}^{n}$. The following are some examples of $n$-manifolds: Euclidean space $\mathbb{R}^{n}$, the unit sphere

$$
S^{n}=\left\{x \in \mathbb{R}^{n+1} \mid\|x\|=1\right\}
$$

in $\mathbb{R}^{n+1}$, the real projective space $P^{n}=\left\{\{x,-x\} \mid x \in S^{n}\right\}$ (with topology on $P^{n}$ defined by $U \subseteq P^{n}$ is open if the union of the family $U$ is open in $S^{n}$, see [3]). If $X$ is an $n$-manifold and $Y$ is an $m$-manifold, then $X \times Y$ is an $n \cdot m$-manifold. Therefore, the space $S^{1} \times \cdots \times S^{1}$ ( $n$-times) is an $n$-dimensional manifold. The torus is the space $S^{1} \times S^{1}$, hence it is a 2-manifold.

A nonempty topological space $X$ is said to be an $n$-manifold with boundary if each point of $X$ has a neighborhood which is homeomorphic to an open subset of $\mathbb{H}^{n}$. If $X$ is an $n$-manifold with boundary, then the subset of $X$ consisting of all points $x \in X$ with the property that there exists a neighborhood $N$ of $x$, an open subset $U$ of $\mathbb{H}^{n}$ and a homeomorphism $f: N \rightarrow U$ such that $f(x) \in \operatorname{Bd} \mathbb{H}^{n}$ is called the boundary of $X$. We denote the boundary of $X$ by $\partial X$. It can be shown (see [10]) that if $x \in \partial X$, then every homeomorphism between a neighborhood of $x$ in $X$ and an open subset of $\mathbb{H}^{n}$ maps $x$ to a point in $\mathrm{Bd} \mathbb{H}^{n}$. It is interesting to remark here that if a point in some topological space has neighborhoods $N_{1}$ and $N_{2}$ which are homeomorphic to open subsets of $\mathbb{H}^{n}$ and $\mathbb{H}^{m}$ 
respectively, then $n=m$; this is a consequence of Invariance of domain theorem, see [10]. In particular, the number $n$, called the dimension of $X$, is uniquely determined by $X$.

If $U$ is an open set in $\mathbb{H}^{n}$ and $z \in U$ is a point such that $z \in \mathbb{H}^{n} \backslash\left(\mathrm{Bd} \mathbb{H}^{n}\right)$, then there exists an open ball in $\mathbb{R}^{n}$ centered at $x$ which is contained in $U$. Furthermore, each open ball in $\mathbb{R}^{n}$ is homeomorphic to $\mathbb{R}^{n}$. Therefore, if $X$ is an $n$-manifold with boundary and $x \in X \backslash \partial X$, then $x$ has an open neighborhood in $X$ homeomorphic to $\mathbb{R}^{n}$. On the other hand, each open ball in $\mathbb{H}^{n}$ centered at a point in $\mathrm{Bd} \mathbb{H}^{n}$ is homeomorphic to $\mathbb{H}^{n}$. It follows from this that each point in $\partial X$ has an open neighborhood in $X$ homeomorphic to $\mathbb{H}^{n}$.

If $X$ is a manifold, then clearly $X$ is a manifold with boundary and $\partial X=\emptyset$. In fact, if $X$ is a manifold with boundary, then $X$ is a manifold if and only if $\partial X=\emptyset$.

If $X$ is a manifold with boundary and $Y$ is homeomorphic to $X$, then clearly $Y$ is also a manifold with boundary and $\partial Y=f(\partial X)$, where $f: X \rightarrow Y$ is a homeomorphism. Furthermore, if $X$ and $Y$ are manifolds with boundary, then $X \times Y$ is a manifold with boundary and the point $(x, y)$ is in its boundary if and only if $x \in \partial X$ or $y \in \partial Y$.

It is not hard to check (see [10]) that the closed unit ball $B^{n}$ in $\mathbb{R}^{n}$ is a manifold with boundary and $\partial B^{n}=S^{n-1}$. For example $[0,1]$ is a manifold with boundary and $\partial[0,1]=\{0,1\}$. Therefore, since $S^{1}$ is a manifold, $S^{1} \times[0,1]$ is a manifold with boundary and $\partial\left(S^{1} \times[0,1]\right)=S^{1} \times\{0,1\}$. (Note that the topological boundary of $S^{1} \times[0,1]$ in $\mathbb{R}^{3}$ differs from $\partial\left(S^{1} \times[0,1]\right)$.)

Theorem 6.1. Let $(X, d, \alpha)$ be a computable metric space and let $S \subseteq X$. Suppose $S$, as a subspace of $(X, d)$, is a manifold with boundary and suppose $S$ and $\partial S$ are semi-computable compact sets in $(X, d, \alpha)$. Then $S$ is a computable compact set in $(X, d, \alpha)$.

Proof. Let $n \geq 1$ be such that $S$ is an $n$-manifold with boundary. If $x \in S \backslash \partial S$, then $x$ has a neighborhood homeomorphic to $\mathbb{R}^{n}$ and by Theorem 5.6 there exists a neighborhood of $x$ in $S$ which is computable up to $S$. On the other hand, if $x \in \partial S$, then there exists an open neighborhood $U$ of $x$ in $S$ and a homeomorphism $f: \mathbb{H}^{n} \rightarrow U$. It is clear that $f\left(\operatorname{Bd} \mathbb{H}^{n}\right) \subseteq$ $U \cap \partial S$ and since for each $z \in \partial S$ every homeomorphism between a neighborhood of $z$ in $S$ and an open subset of $\mathbb{H}^{n}$ maps $z$ to a point in $\mathrm{Bd} \mathbb{H}^{n}$, we have $f\left(\mathrm{Bd} \mathbb{H}^{n}\right)=U \cap \partial S$. It follows from Theorem 5.7 that $x$ has a neighborhood in $S$ which is computable up to $S$.

Hence each $x \in S$ has a neighborhood $N_{x}$ in $S$ which is computable up to $S$. Let $\mathcal{U}=\left\{\operatorname{Int}_{S} N_{x} \mid x \in S\right\}$. Then $\mathcal{U}$ is an open cover of $S$. Since $S$ is compact, there exists a finite subcover of $\mathcal{U}$ for $S$, hence there exist $x_{1}, \ldots, x_{k} \in S$ such that $S=N_{x_{1}} \cup \cdots \cup N_{x_{k}}$. By Proposition $4.2 S$ is a computable compact set.

Corollary 6.2. Let $(X, d, \alpha)$ be a computable metric space and let $S$ be a semi-computable compact set in this space which is a manifold with boundary. Then the following implication holds:

$$
\partial S \text { computable compact set } \Rightarrow S \text { computable compact set. }
$$

Theorem 6.3. Let $(X, d, \alpha)$ be a computable metric space. Let $S$ be a semi-computable compact set in this space such that $S$, as a subspace of $(X, d)$, is a manifold. Then $S$ is computable compact in $(X, d, \alpha)$.

In general, if $X$ is an $n$-manifold with boundary and $\partial X \neq \emptyset$, then $\partial X$ is an $(n-$ $1)$ - manifold. Therefore if $S$ is a manifold with boundary in $(X, d, \alpha)$, then $\partial S$ is a manifold (boundaryless) and we have, by Theorem 6.3, that $\partial S$ is semi-computable compact if and only if $\partial S$ is computable compact. This means that Corollary 6.2 is an equivalent formulation of Theorem 6.1. 
Theorem 6.4. Let $(X, d, \alpha)$ be a computable metric space which is locally computable. Let $S$ be a co-computably enumerable closed set in this space such that $S$, as a subspace of $(X, d)$, is a compact manifold with boundary. Then the following implication holds:

$\partial S$ computable closed set $\Rightarrow S$ computable closed set.

6.1. Application: regular level sets. Suppose that $f: \mathbb{R}^{n} \rightarrow \mathbb{R}^{m}$ is a function of class $\mathrm{C}^{1}$ such that $y \in \mathbb{R}^{m}$ is a regular value of $f$, which means that the differential $D(f)(x)$ : $\mathbb{R}^{n} \rightarrow \mathbb{R}^{m}$ of $f$ in $x$ is surjective for each $x \in f^{-1}\{y\}$. Suppose $f^{-1}\{y\} \neq \emptyset$. Then it is known from differential topology (see [5]) that $f^{-1}\{y\}$ is an $(n-m)$-manifold.

Suppose now additionally that $f$ is computable and $f^{-1}\{y\}$ is a bounded set. In general, if $g: \mathbb{R}^{n} \rightarrow \mathbb{R}$ is a computable function, then $g^{-1}\{0\}$ is a co-c.e. closed set in $\mathbb{R}^{n}$. Therefore $f^{-1}\{y\}$ is a co-c.e. closed set and, by Theorem 6.4. we conclude the following.

Corollary 6.5. If $f: \mathbb{R}^{n} \rightarrow \mathbb{R}^{m}$ is a computable function of class $C^{1}$ and $y \in \mathbb{R}^{m}$ a regular value of $f$ such that $f^{-1}\{y\}$ is bounded, then the set $f^{-1}\{y\}$ is computable.

Example 6.6. (i) Let $S$ be the set of all $(x, y, z) \in \mathbb{R}^{3}$ such that

$$
x^{2}\left(1+e^{x}\right)+y^{2}\left(1+e^{y}\right)+z^{2}\left(1+e^{z}\right)=1 .
$$

We have $S=f^{-1}\{1\}$, where $f: \mathbb{R}^{3} \rightarrow \mathbb{R}$ is defined in the obvious way. Since $\nabla f(x)=$ $\left(\partial_{1} f(x), \partial_{2} f(x), \partial_{3} f(x)\right)=0$ only for the point $x=0$ which is not in $f^{-1}\{1\}$, we have that 1 is a regular value for $f$. Hence $S$ is computable.

(ii) Let $S$ be the set of all $\left(x_{1}, x_{2}, x_{3}, x_{4}\right) \in \mathbb{R}^{4}$ such that

$$
x_{1}^{2}+x_{2}^{2}+x_{3}^{2}+x_{4}^{2}=1
$$

and

$$
\sin x_{1}+\sin x_{2}+\sin x_{3}+\sin x_{4}=1
$$

The intersection of two computable sets need not be computable in general and therefore we cannot get that $S$ is computable only by proving that the sets defined by above equations are computable. We have $S=f^{-1}\{(1,1)\}$, where $f: \mathbb{R}^{3} \rightarrow \mathbb{R}^{2}$ is defined in the obvious way. Let $f_{1}, f_{2}$ be the component functions of $f$. It is easy to check that the vectors $\nabla f_{1}(x)$ and $\nabla f_{2}(x)$ are linearly independent for each $x \in S$. By Corollary $6.5 S$ is computable.

\section{ACKNOWLEDGEMENTS}

The author would like to thank the anonymous referees for their careful work.

\section{REFERENCES}

[1] Vasco Brattka. Plottable real number functions and the computable graph theorem. SIAM J. Comput., 38(1):303-328, 2008.

[2] Vasco Brattka and Gero Presser. Computability on subsets of metric spaces. Theoretical Computer Science, 305:43-76, 2003.

[3] Charles O. Christenson and William L. Voxman. Aspects of Topology. Marcel Dekker, Inc., New York, 1977.

[4] Ryszard Engelking. Dimension Theory. PWN - Polish Scientific Publishers, Warszawa, 1978.

[5] Victor Guillemin and Alan Pollack. Differential Topology. Prentice-Hall, Inc., Englewood Cliffs, New Jersey 
[6] Zvonko Iljazović. Chainable and Circularly Chainable Co-c.e. Sets in Computable Metric Spaces. Journal of Universal Computer Science, 15(6):1206-1235, 2009.

[7] Zvonko Iljazović. Co-c.e. Spheres and Cells in Computable Metric Spaces Logical Methods in Computer Science, Vol. 7(3:05):1-21, 2011.

[8] Takayuki Kihara. Incomputability of Simply Connected Planar Continua Computability, 1(2):131-152, 2012.

[9] Joseph S. Miller. Effectiveness for Embedded Spheres and Balls. Electronic Notes in Theoretical Computer Science, 66:127-138, 2002.

[10] James R. Munkres. Elements of Algebraic Topology. Addison-Wesley Publishing Company, Menlo Park, California, 1984.

[11] Stéphane Le Roux and Martin Ziegler. Singular coverings and non-uniform notions of closed set computability Math. Log. Q., 54:545-560, 2008.

[12] Ernst Specker. Der Satz vom Maximum in der rekursiven Analysis. Constructivity in Mathematics (A. Heyting, ed.). North Holland Publ. Comp., Amsterdam, 254-265, 1959.

[13] Marian B. Pour-El, Jonathan I. Richards, Computability in Analysis and Physics Springer-Verlag, Berlin-Heielberg-New York, 1989.

[14] Alan M. Turing. On computable numbers, with an application to the Entscheidungsproblem Proc. London Math. Soc., 42:230-265, 1936.

[15] Klaus Weihrauch. Computable Analysis Springer, Berlin, 2000. 